\title{
Determination of optical and microphysical properties of thin warm clouds using ground based hyper-spectral analysis
}

\author{
E. Hirsch ${ }^{1}$, E. Agassi ${ }^{2}$, and I. Koren ${ }^{1}$ \\ ${ }^{1}$ Department of Environmental Sciences, Weizmann Institute, Rehovot, 76100, Israel \\ ${ }^{2}$ Department of Environmental Physics, Israel Institute for Biological Research, Nes-Ziona, 74100, Israel
}

Correspondence to: E. Hirsch (eitan.hirsch@weizmann.ac.il)

Received: 2 November 2011 - Published in Atmos. Meas. Tech. Discuss.: 12 December 2011

Revised: 13 March 2012 - Accepted: 12 April 2012 - Published: 27 April 2012

\begin{abstract}
Clouds play a critical role in the Earth's radiative budget as they modulate the atmosphere by reflecting shortwave solar radiation and absorbing long wave IR radiation emitted by the Earth's surface. Although extensively studied for decades, cloud modelling in global circulation models is far from adequate, mostly due to insufficient spatial resolution of the circulation models. In addition, measurements of cloud properties still need improvement, since the vast majority of remote sensing techniques are focused in relatively large, thick clouds. In this study, we utilize ground based hyperspectral measurements and analysis to explore very thin water clouds. These clouds are characterized by liquid water path (LWP) that spans from as high as $\sim 50 \mathrm{~g} \mathrm{~m}^{-2}$ and down to $65 \mathrm{mg} \mathrm{m}^{-2}$ with a minimum of about 0.01 visible optical depth. The retrieval methodology relies on three elements: a detailed radiative transfer calculations in the longwave IR regime, signal enhancement by subtraction of a clear sky reference, and spectral matching method which exploits fine spectral differences between water droplets of different radii. A detailed description of the theoretical basis for the retrieval technique is provided along with a comprehensive discussion regarding its limitations. The proposed methodology was validated in a controlled experiment where artificial clouds were sprayed and their effective radii were both measured and retrieved simultaneously. This methodology can be used in several ways: (1) the frequency and optical properties of very thin water clouds can be studied more precisely in order to evaluate their total radiative forcing on the Earth's radiation budget. (2) The unique optical properties of the inter-region between clouds (clouds" "twilight zone") can be studied in order to more rigorously understanding of the governing physical processes which dominate this region.
\end{abstract}

(3) Since the optical thickness of a developed cloud gradually decreases towards its edges, the proposed methodology can be used to study the spatial microphysical behaviour of these edges. (4) A spatial-temporal analysis can be used to study mixing processes in clouds' entrainment zone.

\section{Introduction}

Clouds play a critical role in the Earth's radiative budget as they modulate the atmosphere by reflecting shortwave solar radiation and absorbing longwave IR (LWIR) radiation emitted by the Earth's surface (Ramanathan et al., 1989). The radiative forcing of a single cloud depends on its height, thickness, and optical properties (Trenberth et al., 2009), and their accumulated effect depends on their lifetime, global coverage, and frequency of occurrence (Wylie et al., 2005). Shallow clouds are usually considered to have a net negative radiative forcing (cooling effect) as they reflect a substantial amount of the received solar irradiance while they have a minor effect on the total thermal radiation emitted by the Earth, since the magnitude of their emitted radiation is comparable with a cloud free surface. On the other hand, high clouds, such as anvils (Koren et al., 2010) or cirrus, might have a positive radiative forcing, since they have a relatively low reflectance in the visible portion of the spectrum, while the thermal contrast between them and the Earth is large. The effect of clouds on the Earth's radiative budget is so profound, that it has been shown that an increase of $15-20 \%$ in the amount of low clouds could balance the expected heating effect of doubling the $\mathrm{CO}_{2}$ concentration in the atmosphere (Slingo, 1990). 
Although extensively studied for decades, cloud modelling in global circulation models (GCM) is far from adequate (Tselioudis and Jakob, 2002), mostly due to insufficient spatial resolution of the circulation models, which results in poor representation of the small scale microphysical processes that control clouds' properties (Heintzenberg and Charlson, 2009). Furthermore, cloud sensing techniques also need improvement (Turner et al., 2007), since most of the techniques which have been developed are oriented to relatively thick clouds that can be easily observed from space-borne platforms. Over the years, numerous algorithms have been suggested to retrieve clouds' properties by remote sensing measurements. These algorithms exploit radiative information in wavelengths from nanometeres to millimeters and can be characterized by the observation point - space or ground based measurements, and by the sensing method - either active or passive.

Although different approaches have been proposed, all of these methods are similar to some extent: they depend upon radiative (e.g. optical thickness and albedo) and microphysical (e.g. droplet size distribution and thermodynamic phase) properties of the clouds. The theoretical basis for determining the optical thickness and effective radius of clouds by using reflected solar radiation was introduced by Nakajima and King (1990). Later, the Special Sensor Microwave/Imager (SSM/I) was used to retrieve liquid water content of clouds over the ocean (Weng and Grody, 1994). In addition, it was suggested that clouds liquid water and temperature could be derived by using an array of ground based devices, namely, microwave radiometer, ceilometer, radio acoustic sounding system and various surface meteorological instruments (Han and Westwater, 1995). The utility of space-borne multispectral passive remote sensing for retrieval of optical and microphysical properties for numerous cloud types was demonstrated (King et al., 1997). On the other hand, ground based cloud radars were used to retrieve the droplets effective radius profile of stratus clouds, by analyzing the radar reflectivity under various assumptions regarding the total number of droplets in the clouds (Frisch et al., 2002). More recently, the passive MODIS Airborne Simulator (MAS) was used to extract cloud properties even over snow and icy backgrounds (King et al., 2004). A noteworthy method to retrieve thin cloud's properties was presented by Turner (2005), who utilized ground based IR high spectral resolution measurements to retrieve mixed-phase cloud properties. The method successfully obtained the properties of simulated and measured thin water clouds with visible optical depths in the range of 0.2-4. The retrieval is based on an optimal estimation approach to fit a measured spectrum to a spectral database that was created by a radiation transfer model. This spectral analysis paradigm, is widely used in retrieval algorithms which exploit multispectral analysis.

As stated above, the main interest of the majority of the remote sensing techniques are relatively large, optically thick clouds, which contribute substantially to the total clouds forcing and to the Earth's albedo. However, Turner et al. (2007) have overviewed the importance of thin liquid water clouds and the challenge in correctly retrieving their properties. In their work, they have defined thin clouds as clouds with liquid water path (LWP) up to $100 \mathrm{~g} \mathrm{~m}^{-2}$ and have compared the performance of almost 20 different algorithms in retrieving the properties of a single layer, overcast, stratocumulus cloud field. The remote sensing techniques considered were wide ranging and spanned from the visible to the microwave portion of the spectrum; from ground based to space borne; both passive and active. The authors' main conclusion was that huge discrepancies exist among the different algorithms (even among algorithms that are in the same general classification), and that the cloud properties retrieval community should examine the accuracy of the algorithms when thin clouds are considered. An additional complexity to the challenges in correctly retrieving thin clouds properties arises from their expected occurrences. Koren et al. (2008) have studied the dependence of clouds' frequency (in a sparse cumulus cloud field) on their apparent size and showed that a power law governs the connection. This means that the number of clouds with a given area is proportional to the inverse of $a m$, where $a$ is the cloud area and $m$ is the power exponent of the distribution $(1<m<2)$. They concluded that the smallest detectable clouds may contribute significantly to the total cloud area as well as for the mean cloud field reflectance. This analysis coincides well with studies (Koren et al., 2007) which identified optically unique properties of the clouds' inter region (also known as 'the clouds' twilight zone"). This region is characterized by a decrease in the apparent optical depth as the distance from the nearest detectable cloud increases. The precise nature of the "twilight zone" is still unclear, although several mechanisms have been suggested to explain its optical properties: undetected cloud fragments, aerosol humidification, and nearby scattering of solar radiation by clouds, are only some of the main suggested explanations. As most of the current retrieval methods are applied to space borne sensor data, thin warm clouds are usually ignored due to insufficient spatial and temporal resolution. In particular, the transition zone between clouds and clear skies is usually classified into one of two possible states: cloudy or clear. In spite of the knowledge gaps regarding the nature of thin, warm clouds, the relationship between their LWP and effective radius was studied. Liu et al. (2003) found high correlation between clouds' LWP and their effective radius: thin, undeveloped clouds, which contain small amounts of LWP, are characterized by small effective radii as well. In fact, Liu et al. (2003) showed that the majority of clouds with LWP smaller than $25 \mathrm{~g} \mathrm{~m}^{-2}$ had effective radius smaller than $5 \mu \mathrm{m}$. Luckily (and as presented later in this manuscript), our proposed method is at its highest quantitative sensitivity for clouds with small effective radii. The purpose of our study is to introduce a new concept to determine and retrieve the effective radius, LWP, and optical depth of very thin warm clouds by using ground based IR spectral 
measurements. These kinds of measurements and analysis might shed light on some of the most interesting enigmas of cloud microphysics and interaction with aerosols, and the clouds' effect on the radiative budget of the Earth.

In this study, we will utilize ground based infrared spectral analysis and measurements in order to explore very thin water clouds which are characterized by liquid water path that varies from as high as $50 \mathrm{~g} \mathrm{~m}^{-2}$ and down to $65 \mathrm{mg} \mathrm{m}^{-2}$ with a minimum of approximately 0.01 visible optical depth. The following section will introduce the theoretical basis for ground based passive sensing of the thermal IR spectrum of the zenith sky. The retrievals for the cloud effective radius and liquid water path (and the optical depth as a result) will be developed. The spectral differences in the extinction efficiencies of water droplets with different radii will be examined and the effect of these differences on the radiative transfer calculations will be discussed. We will show that thin liquid water clouds which contain varying liquid water paths and effective radii alter the magnitude and the spectrum of the downwelling IR sky radiation. We will demonstrate how adequate spectral analysis can serve as a trigger for a mathematical best fit retrieval algorithm. This concept results in a standalone algorithm which first spectrally identifies the physical phenomenon which formed the measured signal, and only then is the optimal solution chosen in terms of mathematical best fit. The limitations of the method are discussed in Sect. 3 by an overview of the methodology's sensitivity to fluctuations in the relative humidity field, atmospheric aerosols, and haze. Section 4 describes the experimental setup used to validate the proposed methodology, while Sect. 5 presents a case study of a natural cloud that was measured during a field campaign. The main conclusions of these results and future work are discussed in detail in Sect. 6.

\section{The downwelling radiance of the zenith sky in the presence of thin warm clouds in the long wave IR spectral band}

In this section we will explore the theoretical basis for the spectral variability of the zenith sky radiance in the LWIR, in the presence of thin warm clouds. We will show that clouds which differ by their effective droplets radii alter the downwelling sky radiance in a manner that can be identified by high spectral resolution measurements. Nevertheless, these spectral differences are small when compared to the clear sky background, and therefore a differential analysis technique is required in order to extract them from the clear sky background signature. As will be presented, this technique exploits the advantages of ground based, high temporal resolution measurements.

We will first explore how the obtained spectral properties of a single water droplet varies with its radius. Then a brief introduction to the analytical methods which have been used

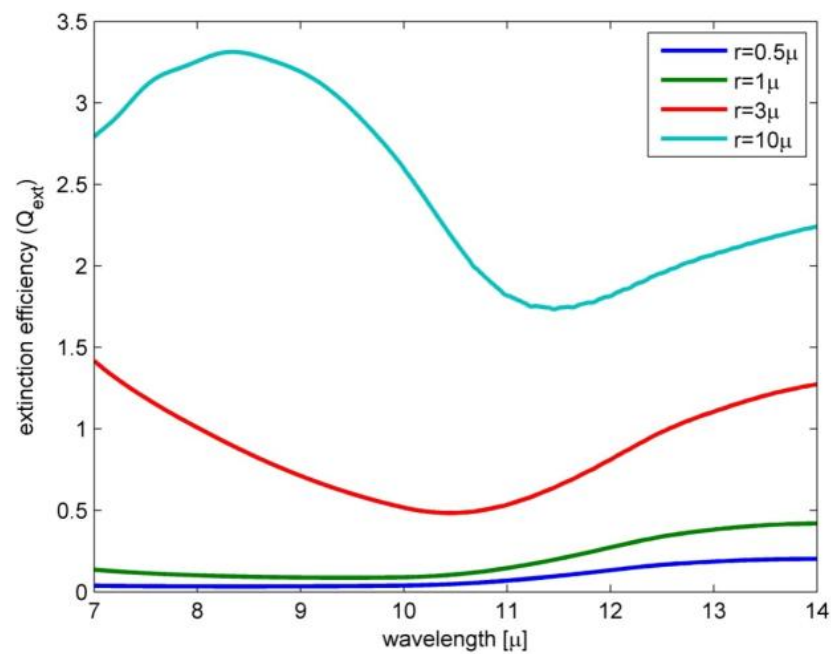

Fig. 1. Extinction efficiency vs. wavelength for water droplets at different radii. Note the evident spectral difference of the various droplets. This analysis suggests that thin water clouds which differ by their droplet's effective radius, might have a unique spectrum in the LWIR. As a result, it might be possible to discriminate between water droplets of different sizes.

in this study is provided. Following that, we analyze the effect of thin monodisperse and polydisperse water clouds on the measured sky LWIR radiance. Finally, at the end of this section, we provide a detailed scheme of a retrieval method for the microphysical and the optical properties of very thin water clouds. This method is based on a spectral comparison between measured spectra and a spectral library created by radiative transfer calculations.

\subsection{The spectral dependence of the extinction efficiency on the droplet's radius}

The presence of water droplets in the atmosphere affects the magnitude and the spectrum of the LWIR sky radiation that reach the surface. The interaction of the radiation with the droplets is described by the generalized Mie (1908) theory, which derives a wave equation with boundary conditions at the surface of a sphere by solving Maxwell's equations. The extinction, absorption, and scattering efficiencies are commonly characterized as a function of the size parameter (which is defined as $x=2 \pi r / \lambda$, where $r$ is the droplet's radius and $\lambda$ is the radiation wavelength). An alternative way to characterize the interaction of radiation and water droplets is to examine the mentioned efficiencies in a certain spectral region (e.g. the LWIR), for different droplets' radii. Such analysis reveals variations in the extinction efficiencies of droplets with different radii (Fig. 1). The refractive index of water (Palik, 1997), was used in order to calculate the extinction, absorption, and scattering efficiencies (Mätzler, 2002). The scattering coefficient is larger than the absorption coefficient (per unit length), therefore scattering is the dominant 
mechanism in which water attenuates the impinging LWIR radiation. Apart from the evident differences in the magnitude of the extinction efficiency, one can notice how the extinction spectrum depends on the droplets radii. These spectral variations suggest that thin warm clouds with different droplets' effective radii might modify the down-welling sky radiance in distinct spectral forms. In the following subsections we will examine the latter hypothesis, by using a radiative transfer model which incorporates the optical properties of water clouds containing different water droplet size distributions.

\subsection{Analysis methodology}

The attempt to extract physical properties of remotely sensed objects is commonly referred to as solving an inverse problem. A typical approach to apply it is composed of a forward model that predicts the expected signal under certain atmospheric parameters, and a mathematical curve fitting technique and threshold criteria to decide which atmospheric parameters present the most probable solution (Rodgers, 2000). As detailed hereafter, the presented methodology follows this general approach but with important modification prior to the stage of curve fitting technique. The presented approach treats thin warm clouds as semi transparent objects which alter the magnitude and the spectrum of the clear background signal. Therefore, we have used techniques borrowed from the field of remote detection and identification of gaseous and aerosols plumes for environmental applications, under the assumption that they are the most suitable tools to retrieve thin clouds' properties (see for example, Hirsch and Agassi, 2007, and Agassi et al., 2008).

The analysis presented in this study relies on 3 elements: a radiation transfer model, a method to extract the effect of a water cloud while eliminating the sky background, and a spectral matching method. A brief overview of each element is given in the following subsections.

\subsubsection{Radiative transfer model}

In this study, the PCModWin4.0 software, which includes the MODTRAN model (Berk et al., 1989), was used to predict the expected zenith sky radiance in the presence of various thin water clouds. MODTRAN is a radiation transfer model developed by the US Air Force Research Laboratory. It is based on the HITRAN2000 database (Rothman et al., 2003) and it solves the radiative transfer equation including the effects of molecular and particulate absorption/emission and scattering, surface reflections and emission, solar/lunar illumination, and spherical refraction. The atmosphere is modeled as plane parallel (horizontally homogeneous), and its constituent profiles, both molecular and particulate, may be defined by using built-in models or by user-specified vertical profiles. The spectral range extends from the UV into the farinfrared and the user can define up to 4 aerosol types with distinct optical properties and incorporate each aerosol in the atmospheric column with specific extinction, absorption, and scattering coefficients. In our analysis, the MODTRAN input included the sounded atmospheric profile, the vertical extent of the cloud, and its water droplets' optical properties which were calculated for every simulated cloud.

\subsubsection{Signal enhancement by sky background elimination}

The presence of a thin water cloud alters the clear sky IR radiation. Unfortunately, as demonstrated in Sect. 2.3 below, the magnitude of the signal contributed only by the thin cloud is very small compared to the signal emerging from the clear sky itself. Therefore, in order to enhance the cloud's signature, we subtract the clear sky signal from the obtained spectra. The method of clear reference subtraction is commonly applied for detection and identification of weak gaseous plumes (Hirsch and Agassi, 2007). It enables retrieval of only the differential spectral signal, and therefore analyzes the phenomenon which created the signal without considering the background. This method is particularly advantageous for weak semi-transparent objects that otherwise could not be analyzed, and in the following we will show how apparent similar spectra become spectrally distinctive when the clear sky reference is removed.

\subsubsection{Spectral analysis}

The process of subtracting the clear sky reference creates a differential spectrum. In order to classify the phenomenon which caused the differential signal, we assess its spectral similarity with a predicted set of library spectra which was pre-calculated. As commonly applied for identification of known reference spectra (Yuhas et al., 1992; Park et al., 2007), we have utilized the SAM - Spectral Angle Mapping (Kruse et al., 1993) analysis on the differential spectral signatures (Fig. 2). The SAM considers the spectral signals (each consisting of $n$ wavelengths) as vectors in a $n$-dimensional space, and calculates the angle between two spectra as a measure of their similarity. The angle between two spectra can be any value between $0^{\circ}$ (perfect match), through $90^{\circ}$ (orthogonal spectra), to $180^{\circ}$ (two spectra pointing to opposite directions). Namely, distinct spectra will produce relatively large SAM values, while similar spectra will produce small values. As in any remote sensing retrieval, a certain threshold must be applied, and its value depends on the application and should be determined empirically. In the proposed method, and as described later, we have applied a SAM threshold of $10^{\circ}$. The SAM metric is especially efficient when small spectral features are present, even though the complete spectral behaviour appears similar. Throughout this study, the SAM method has been applied on spectra which contained 16 wavelengths in the region of $8-9 \mu \mathrm{m}$ and 51 wavelengths in the region of $10-13 \mu \mathrm{m}$. These wavelengths were chosen to 


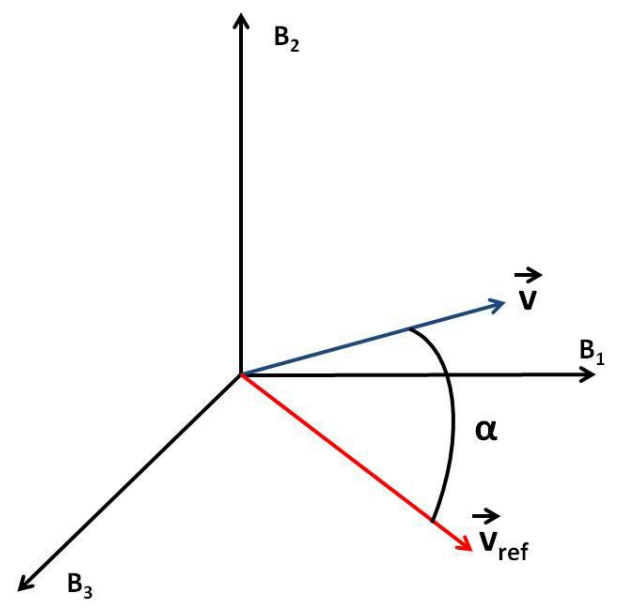

Fig. 2. An example of the SAM (Spectral Angle Mapping) index of two spectra. SAM considers a measured spectrum in $n$ wavelengths ( $n=3$ in this example) as a vector in an $n$ dimensional space $\left(B_{1}, B_{2}, B_{3}\right)$. The spectral similarity between a measured spectrum $(V)$ and a reference spectrum $\left(V_{\text {ref }}\right)$ is simply the angle $\alpha$ between these two spectra, calculated to be $\alpha=\cos ^{-1}\left(\frac{v v_{\text {ref }}}{\|v\| \cdot\left\|v_{\text {ref }}\right\|}\right)$. Distinct spectra will produce relatively large SAM values, while similar spectra will produce small values.

match with the spectro-radiometer features used in our measurements (Sect. 4). The spectral region of $9-10 \mu \mathrm{m}$ was deliberately omitted since it contains the wide ozone absorption line (McCaa and Shaw, 1968), that might induce errors in the analysis.

\subsection{The LWIR sky radiance in the presence of very thin warm monodisperse and polydisperse clouds}

In this subsection we will investigate the spectral effect of very thin water clouds on the down welling IR radiance of the clear sky. Throughout this study we will use the atmospheric profile measured with a radiosonde in the Beit-Dagan station (http://weather.uwyo.edu/upperair/sounding.html), on $8 \mathrm{Au}$ gust 2010, 12:00 UTC (Fig. 3), as an input for the radiative transfer calculations. During the Israeli summer, there is a steady downwelling warm air settling over the Middle East that originates from the tropics. Therefore, a strong inversion layer is present, and only very shallow clouds can form. The occurrence of precipitation is almost impossible. In addition, the all area is dominated by steady westerly winds which arise from a constant low pressure over the Persian Gulf. This low pressure itself is a branch of the Indian summer monsoon. Since the Israeli summer is characterized by inactive weather and very stable meteorological conditions, the chosen atmospheric profile represents a typical Israeli daytime summer profile. According to the ceilometer readings in the Ben-Gurion airport (http://weather.uwyo.edu/surface/ meteogram/), which is located approximately $10 \mathrm{~km}$ from our measurement site, the average cloud base height was

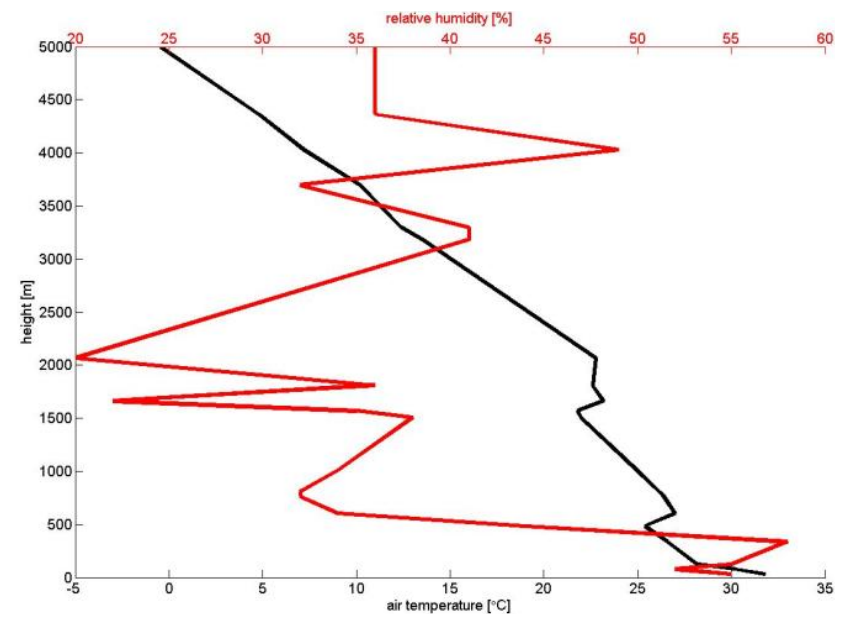

Fig. 3. Atmospheric profile (relative humidity in red, and air temperature in black) on 8 August 2010, 12:00 UTC (http://weather. uwyo.edu/upperair/sounding.html). The shallow inversion layer is noticeable at a height of about $600 \mathrm{~m}$.

$792 \mathrm{~m}$ during the morning hours. Therefore, all the radiative transfer calculations simulated clouds at $800 \mathrm{~m}$ above the surface.

\subsubsection{The effect of monodisperse water clouds on the LWIR sky radiance}

The extinction coefficient per unit length of a monodisperse water cloud with a given liquid water content (LWC) is given by

$\sigma_{\text {ext }}(\lambda)=N \pi r^{2} Q_{\text {ext }}(\lambda, r)$

where $r$ is the droplet radius, and $Q_{\text {ext }}(\lambda, r)$ is the extinction efficiency of a single water droplet with radius $r$ at wavelength $\lambda$. $N$ is the droplet density (i.e. the number of droplets per unit volume) which equals the LWC divided by the mass of a droplet, i.e. $N=\operatorname{LWC} /\left(4 / 3 \pi r^{3} \rho\right.$ ) (where $\rho$ is the density of water, taken here in mks units as $1000 \mathrm{~kg} \mathrm{~m}^{-3}$ ). Exactly the same formulation holds for deriving the absorption and scattering coefficients $\left(\sigma_{\mathrm{abs}}(\lambda)\right.$ and $\sigma_{\text {sct }}(\lambda)$, respectively) per unit length. These spectral coefficients are used to solve the radiative transfer equations in the presence of clouds.

A set of $50 \mathrm{~m}$ thick, monodisperse clouds with equal liquid water content of $15 \mathrm{mg} \mathrm{m}^{-3}$, were simulated at equal heights and under the same atmospheric profile. The only difference between the clouds was the water droplet radius. The expected zenith sky LWIR radiances in the presence of the simulated clouds are presented (Fig. 4). The figure creates an impression that the anticipated spectra are quite similar, since most of the signal originates from the clear sky background. As explained above, subtraction of the clear reference eliminates the sky background and extracts the sole effect of the thin clouds (Fig. 5). The differential spectra appear quite distinct as small droplets attenuate more radiation in the 


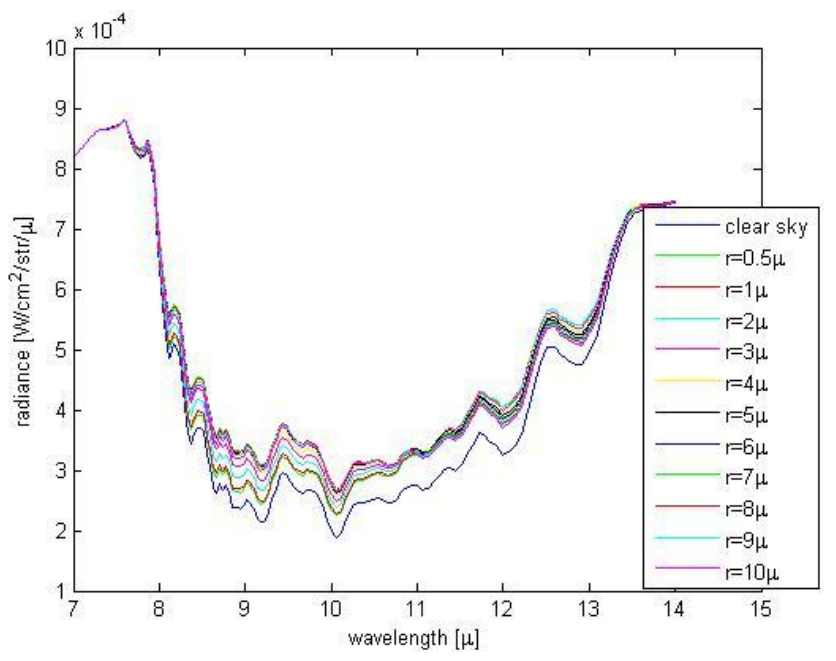

Fig. 4. The expected spectral signature of the zenith sky in the presence of $50 \mathrm{~m}$ thick monodisperse clouds with liquid water content of $15 \mathrm{mg} \mathrm{m}^{-3}$ at $800 \mathrm{~m}$ above the ground. Every cloud is characterized by different water droplets radii. At first glance, the spectral signatures appear extremely similar.

$11-13 \mu \mathrm{m}$ region while large droplets attenuate more radiation in the 8-9 $\mu \mathrm{m}$ region. The use of the SAM index on the differential spectra (Fig. 6) quantifies the apparent spectral variations of the sky radiance in the presence of thin clouds. Three distinctive areas appear in the mutual cross SAM matrix of the differential spectra: (1) small water droplets with radii less than $2 \mu \mathrm{m}$ have spectral signals which are relatively similar (low cross SAM values), while their spectra are distinct from water droplets with higher radii. (2) Medium size water droplets with radii of $2-3 \mu \mathrm{m}$ are different from water droplets with other radii. (3) Spectra of larger water droplets with radii greater than $4 \mu \mathrm{m}$ appear spectrally different than smaller droplets, but the ability of the technique to distinguish between droplets in the region of $4-10 \mu \mathrm{m}$ is relatively low. These distinctive areas reinforce the hypothesis that spectral variations in the extinction efficiencies alter the radiation in a spectrally different manner, and that using the SAM on the differential spectral signatures might be useful to distinguish between clouds with different droplets' effective radii.

\subsubsection{The effect of polydisperse water clouds on the LWIR zenith sky radiance}

Clouds droplets are not monodisperse. The size distribution of water droplets of cumulus clouds has been extensively studied for several decades. A modified Gamma function was proposed to represent the density size distribution of clouds' droplets as a function of their radius (Deirmendjian, 1964). Specifically, the size distribution is given by

$n(r)=a r^{\alpha} e^{-b r \gamma}$

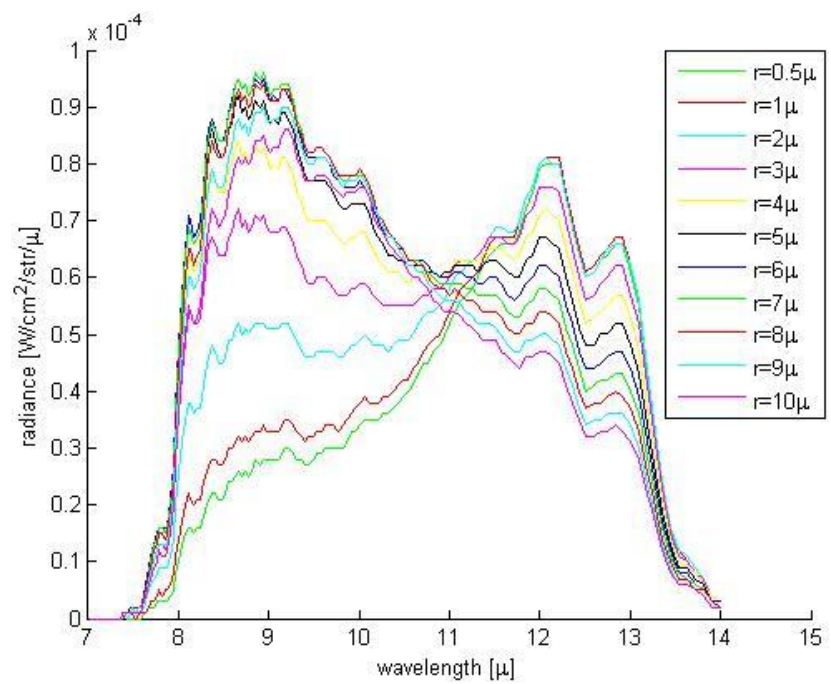

Fig. 5. The differential spectral signature of the various clouds presented in Fig. 4. Every signature in this figure is the result of subtracting the clear sky signature (see Fig. 4) from the expected sky radiance in the presence of a cloud. One can notice the evident spectral difference caused by different water droplets ' radii. Clouds with small water droplets attenuate more radiation in the $11-13 \mu \mathrm{m}$, while clouds with larger droplets affect more in the $8-9 \mu \mathrm{m}$.

where $n(r)$ is the normalized size distribution, i.e. $\int_{0}^{\infty} n(r)$ $\mathrm{d} r=1$, and $a, \alpha, b$, and $\gamma$ are positive constants. Utilizing the modified Gamma size distribution, we have created a large dataset of more than 120000 size distributions of water droplets in order to cover a wide range of possible cases. The droplets size distributions which were used in our simulation (Table 1), represent typical distributions that were measured in natural low clouds (Miles et al., 2000). These simulated distributions span a 2-D space of effective radius $\left(r_{\mathrm{eff}}\right)$ and LWC (Fig. 7), which will later be used by MODTRAN to construct a spectral library of possible sky radiances in the presence of different water clouds. The effective radius is a commonly used parameter to represent the water droplets size distribution (Hansen and Travis, 1974). Specifically,

$r_{\text {eff }}=\frac{\int_{0}^{\infty} r^{3} n(r) \mathrm{d} r}{\int_{0}^{\infty} r^{2} n(r) \mathrm{d} r}$

and it is related to the visible optical depth of the cloud (Stephens, 1994), by

$\mathrm{OD}_{\mathrm{vis}}=\frac{3 \mathrm{LWP}}{2 \rho r_{\mathrm{eff}}}$

where LWP is the liquid water path in the cloud, namely $\mathrm{LWP}=\int_{h_{\text {base }}}^{h_{\text {top }}} \operatorname{LWC}(h) \mathrm{d} h$. 
Table 1. Parameters of clouds droplet size distributions used in our simulation (right column), and as summarized from vast in-situ measurements of droplets size distributions in low-level continental and marine Stratiform clouds (Miles et al., 2000). Values in parenthesis represent the parameter's standard deviation

\begin{tabular}{llll}
\hline Parameter & \multicolumn{2}{c}{$\begin{array}{c}\text { In-situ measurements } \\
\text { (Miles et al., 2000) }\end{array}$} & $\begin{array}{l}\text { Simulated } \\
\text { droplets size } \\
\end{array}$ \\
\cline { 2 - 2 } & Continental & Marine & distributions \\
\hline Mean radius $[\mu \mathrm{m}]$ & $4.1(1.95)$ & $7.1(1.7)$ & $6.3(3.8)$ \\
Standard deviation above mean radius $[\mu \mathrm{m}]$ & $1.55(0.6)$ & $2.9(1)$ & $3.4(2.7)$ \\
Effective radius $[\mu \mathrm{m}]$ & $5.4(2.05)$ & $9.6(2.35)$ & $9.6(6.5)$ \\
LWC $\left[\mathrm{mg} \mathrm{m}^{-3}\right]$ & $190(210)$ & $180(140)$ & $118(128)$ \\
\hline
\end{tabular}

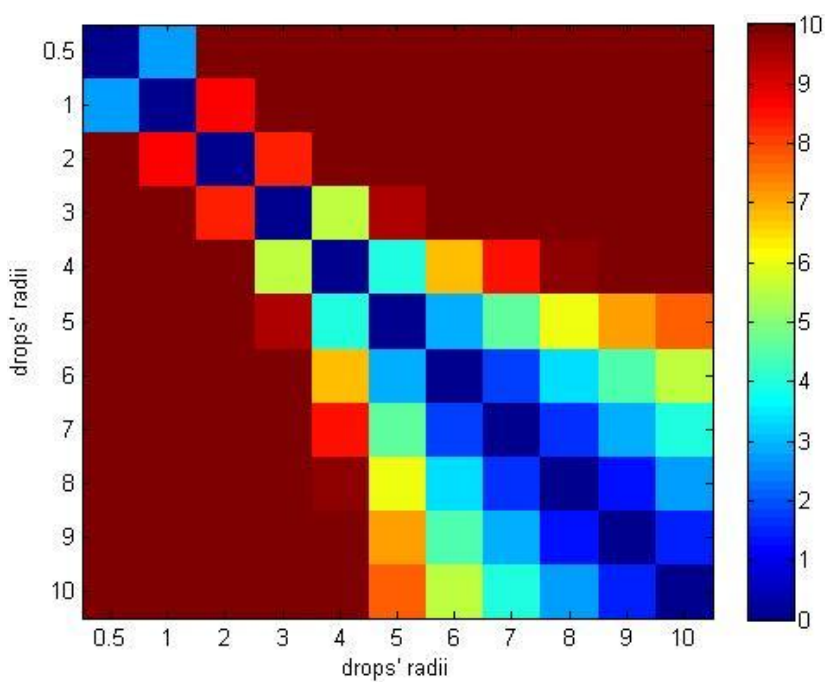

Fig. 6. Cross SAM values of the differential spectral signatures formed by thin clouds (Fig. 5). Small values indicate the signatures are spectrally similar, while large values indicate the signatures are spectrally distinct. Three distinctive areas exist in the matrix: (1) small water droplets with radii less than $2 \mu \mathrm{m}$ create spectral signatures which are relatively similar (low cross SAM values), while their spectral signature is distinctive from water droplets with higher radii. (2) Medium water droplets with radii of $2-3 \mu \mathrm{m}$ are unique from water droplets with other radii. (3) Spectral signatures of larger water droplets with radii higher than $4 \mu \mathrm{m}$ appear spectrally different than smaller droplets, but the method's capability to distinguish between droplets in the region of $4-10 \mu \mathrm{m}$ is relatively low.

It is worth noticing that the use of $r_{\text {eff }}$ as a measure for the cloud optical depth is not valid in the IR region, as the original calculation of $r_{\text {eff }}$ (Hansen and Travis, 1974), assumed the droplet radius is noticeably larger than the wavelength, which is valid only in the visible portion of the spectrum. Nevertheless, in order to follow conventional characterization of clouds in the scientific community, we will use the $r_{\text {eff }}$ and $\mathrm{OD}_{\text {vis }}$ parameters to characterize the simulated and measured clouds.

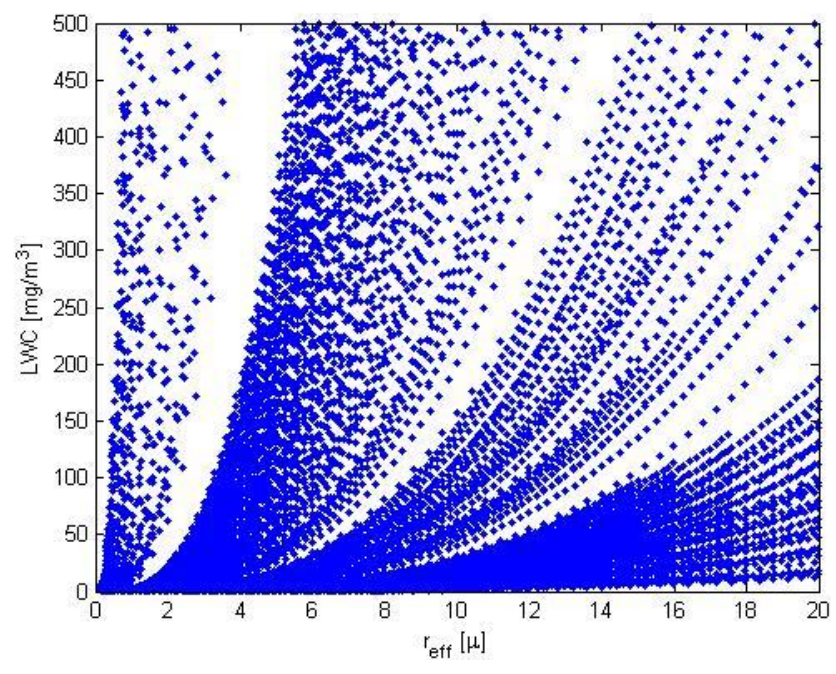

Fig. 7. The space of LWC and effective radius of the different clouds that were used in this analysis. Every point in this graph represents a modified Gamma size distribution with distinct parameters. The size distribution is used to calculate the extinction, absorption, and scattering coefficients for unit length of the simulated cloud.

In a similar manner to Eq. (1), the spectral extinction coefficient of a water cloud with a given droplet size distribution is

$\sigma_{\text {ext }}(\lambda)=N \int_{0}^{\infty} n(r) \pi r^{2} Q_{\text {ext }}(\lambda, r) \mathrm{d} r$

where $N$ is the total number of water droplets per unit volume, and $n(r)$ is a normalized size distribution as defined previously.

The same cross SAM analysis on the differential spectral signatures of polydisperse water clouds (Fig. 8) shows that the spectral variability still holds even when the droplets follow a modified Gamma distribution function. From the figure, it is obvious that clouds with small $r_{\text {eff }}$ (less than $1 \mu \mathrm{m}$ ) are spectrally distinct, as their cross SAM values with other clouds are relatively high. In the same manner, clouds with medium effective radius $\left(1 \mu \mathrm{m}<r_{\text {eff }}<2.75 \mu \mathrm{m}\right)$ are easily 


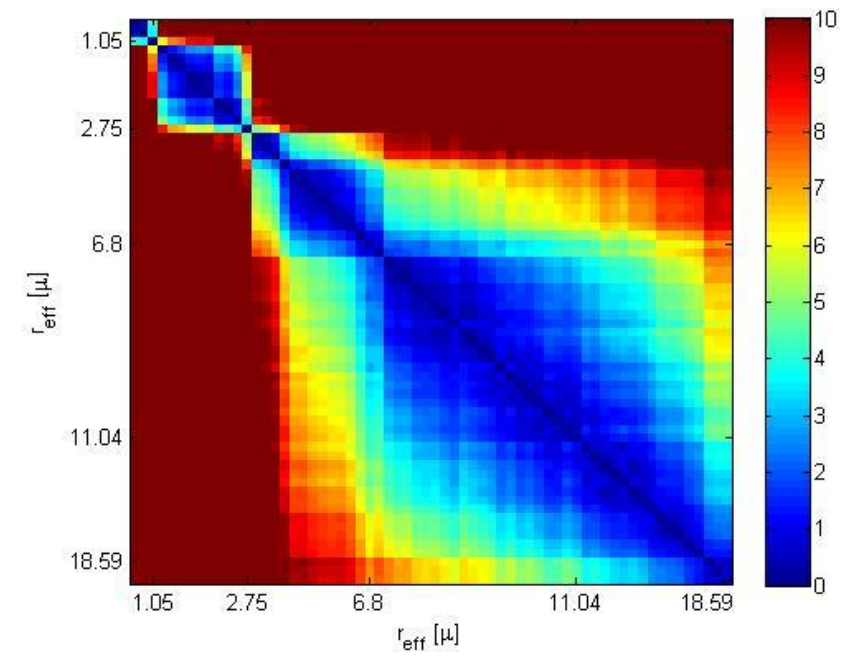

Fig. 8. Cross SAM values of the differential spectral signatures of water clouds with different effective radii with approximately constant LWC $\left(14.5 \mathrm{mg} \mathrm{m}^{-3}<\mathrm{LWC}<15 \mathrm{mg} \mathrm{m}^{-3}\right)$. This figure shows that the spectral variability depends on the effective radius of the cloud: Clouds with small $r_{\text {eff }}$ (less than $1 \mu \mathrm{m}$ ) are spectrally distinct, as their cross SAM values with other clouds are relatively high. Clouds with medium effective radius $\left(1 \mu \mathrm{m}<r_{\text {eff }}<2.75 \mu \mathrm{m}\right)$ are easily distinguished by the SAM analysis. Clouds with $r_{\text {eff }}>3 \mu \mathrm{m}$ appear relatively similar to each other, but quite different from a cloud with smaller $r_{\text {eff }}$.

distinguished by the SAM analysis. Clouds with $r_{\text {eff }}>3 \mu \mathrm{m}$ appear relatively similar to each other, but quite different from clouds with smaller $r_{\text {eff }}$.

\subsection{Retrieval methodology}

Following the theoretical basis presented in the previous subsections, we propose the following methodology (Fig. 9), which is composed of 3 elements, for the retrieval of very thin liquid water clouds' microphysical and optical properties:

a. Simulating the expected IR spectral signature of the zenith sky in the presence of very thin liquid water clouds with various microphysical and geometrical properties, namely effective radius, LWC, and geometrical depth. The usage of these three parameters allows us to refer to the simulated and retrieved clouds by four variables: effective radius, LWC, LWP, and optical depth, and we will use them interchangeably throughout this manuscript according to the scientific relevance. The simulation should use a radiative transfer model, that considers effects of molecular and particulate emission and scattering, and create a large spectra database which will serve as a spectral library.

b. Continuous, ground based, measuring of the zenith sky: in order to analyze small scale dynamical processes

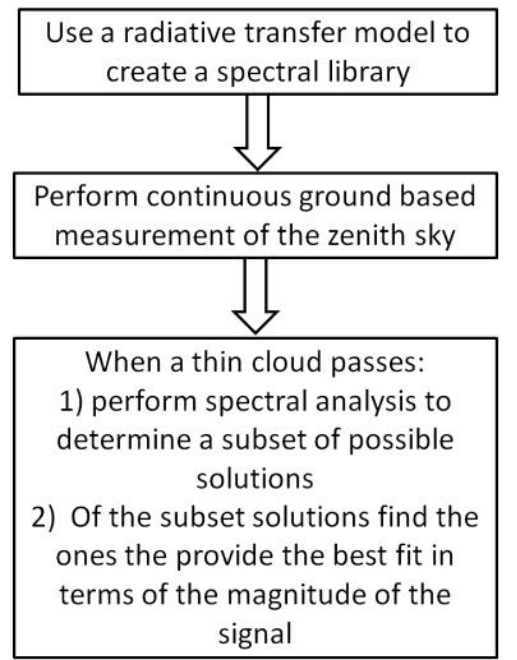

Fig. 9. A flowchart of the proposed methodology for the retrieval of very thin liquid water clouds' microphysical and optical properties.

within the clouds, measurement of the zenith sky with reasonable spectral, spatial, and temporal resolution is needed. The sensor's field of view (FOV) should not exceed several milliradians, which correspond to several square meters at the top of the boundary layer. The acquisition rate should be as high as possible in order to allow the retrieval of the temporal dynamics of the cloud, and the sensor's sensitivity should be high enough to extract the signal formed by the presence of the thin clouds. Regarding the required spectral resolution, the analysis presented in this study utilized 67 spectral bands. Nevertheless, it is possible that the spectral analysis can be performed with a relatively small number of narrow spectral bands, and as suggested in Sect. 3, even 5 spectral bands might be suitable to sustain the spectral variability between thin clouds.

c. Retrieving the microphysical and optical properties of a cloud whenever a substantial increase in the measured sky radiation occurs: using a spectral match index (SAM analysis for example) between the measured signal and the spectral library. This spectral analysis will serve as a trigger for the retrieval process by identifying that the source of the measured signal is indeed water droplets. The second stage is comparing the magnitude of the measured signal with the spectral library signals and choosing a set of possible solutions of liquid water path, effective radius, and optical depth of the cloud.

The results of utilizing the proposed methodology on measured data are presented in Sects. 4 and 5, where a controlled experiment was conducted in order to validate the proposed methodology, and natural clouds were measured and analyzed during a field campaign. 
Table 2. The range of the LWC, LWP, $r_{\text {eff }}$, thickness, and optical depths of the simulated clouds.

\begin{tabular}{cccccc}
\hline $\begin{array}{c}\text { Liquid } \\
\text { water } \\
\text { content } \\
{\left[\mathrm{mg} \mathrm{m}^{-3}\right]}\end{array}$ & $\begin{array}{c}\text { Liquid } \\
\text { water } \\
\text { path }\left[\mathrm{g} \mathrm{m}^{-2}\right]\end{array}$ & $\begin{array}{c}\text { Cloud's } \\
\text { depth }[\mathrm{m}]\end{array}$ & $\begin{array}{c}\text { Effective } \\
\text { radius } \\
{[\mu \mathrm{m}]}\end{array}$ & $\begin{array}{c}\text { Optical } \\
\text { depth } \\
(\lambda=550 \mathrm{~nm})\end{array}$ & $\begin{array}{c}\text { Optical } \\
\text { depth } \\
(\lambda=10 \mu \mathrm{m})\end{array}$ \\
\hline $2.6-500$ & $0.065-49.26$ & $10-100$ & $0.16-20$ & $9.4 \times 10^{-3}-138$ & $8.5 \times 10^{-3}-4.48$ \\
\hline
\end{tabular}

\section{Sensitivity analysis and induced bias or misclassification in the retrieved parameters}

This section presents an analysis of the range of the optical and microphysical properties that can be retrieved by the proposed method. In addition, the method's sensitivity to the number of spectral bands and noise level is analyzed. Moreover, a detailed discussion regarding the bias or misclassification that can be induced as a result of fluctuations in the relative humidity, aerosols, and haze is presented.

\subsection{Sensitivity analysis}

In order to assess the method's range of application we need to use a specific atmospheric profile in our radiative transfer model and to consider the performance in terms of signal to noise ratio (SNR). As before, the atmospheric profile used for this analysis was measured during 8 August 2010 12:00 UTC at a nearby meteorological station (http://weather.uwyo.edu/ upperair/sounding.html). As presented in the following section 4 , a controlled experimental setup was used to validate the proposed method. Our main instrumental device was the SR5000 (CI-Systems, Israel), a calibrated spectro-radiometer in the range of 2.5-14 $\mu \mathrm{m}$ (see Appendix A for further details). The radiometer was calibrated with an extended area infrared source (SR80, CI-Systems, Israel), and the noise equivalent spectral radiance (NESR) level was measured in the lab, and it found to be $6.4 \times 10^{-6} \mathrm{~W} \mathrm{~cm}^{-2} \mathrm{str}^{-1} \mu \mathrm{m}^{-1}$, for a wavelength of $10 \mu \mathrm{m}$. As detailed below, through our analysis a SNR threshold of 3 was applied.

For the mentioned atmospheric profile (see Fig. 3), we used radiative transfer calculations to create a spectral library which included a total of 121010 clouds. This spectral library considers the effect of varying LWC, effective radius, and geometrical thickness of the clouds. In order to model realistic cases of thin clouds that can be measured by our sensors, we have filtered out some of the clouds from the spectral library. On one hand, as a cloud becomes thinner, its optical depth and its effect on the sky radiance diminishes. On the other hand, as a cloud becomes optically thicker, its apparent IR spectral signature approaches the emitted radiance of a blackbody at the same temperature as the air layer in which it resides (Yamamoto et al., 1970). At this limit, the proposed methodology cannot distinguish between signals that originate from thick clouds with different effective radii. As a result, as the simulated clouds gain optical depth, the spectral

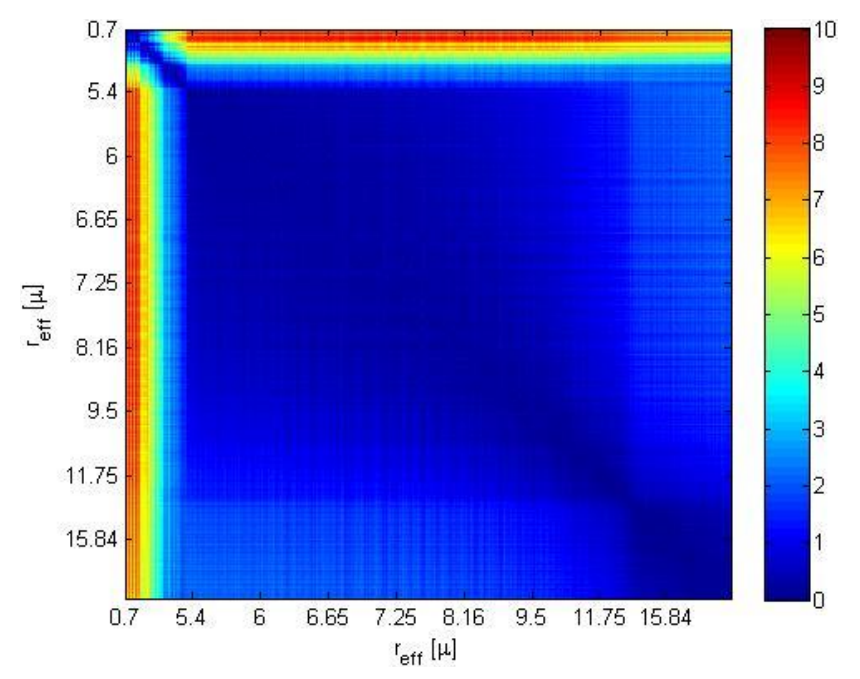

Fig. 10. Cross SAM values of the differential spectral signatures of water clouds with different effective radii with relatively high LWC of $400-500 \mathrm{mg} \mathrm{m}^{-3}$. Comparing this figure to Fig. 8 , we see that the ability to spectrally distinguish clouds with different effective radius has worsened, as a result of the higher LWC values which cause the clouds' spectral signatures to approach the radiation spectrum emitted by a blackbody.

variability decreases gradually, until the point where almost no spectral variation exists whatsoever (Fig. 10).

Let $S$ be the spectrum of the zenith sky in the presence of a thin cloud, and $S_{\text {sky }}$ be the spectrum of the clear zenith sky. Generally, we consider only the clouds with a signal to noise ratio (SNR) larger than 3 at a wavelength of $10 \mu \mathrm{m}$ (compared to the noise level of the SR5000 spectro-radiometer), but are still thin enough (i.e. their obtained signal is distinct from a blackbody spectrum). Specifically, we considered all the clouds which passed the following criteria:

$$
\begin{aligned}
& s_{\lambda=10 \mu}-s_{\text {sky }, \lambda=10 \mu}>3 \times \operatorname{NESR}_{\lambda=10 \mu} \text { and } \\
& \left.\frac{s-s_{\text {sky }}}{s_{\text {sky }}}\right|_{\lambda=10 \mu}<0.9 \times \max _{\text {over_all_clouds }}\left(\left.\frac{s-s_{\text {sky }}}{s_{\text {sky }}}\right|_{\lambda=10 \mu}\right) .
\end{aligned}
$$

The right hand side of the criteria ensures that we filter out all the thick clouds with spectral behaviour similar to a blackbody. The result of these screenings was a database consisting of 81197 spectral signatures with varying parameters (see Table 2). 


\subsubsection{Method's sensitivity to instrumental noise level}

As stated above, we used the SR5000 spectro-radiometer which has a noise level of $6.4 \times 10^{-6} \mathrm{~W} \mathrm{~cm}^{-2} \mathrm{str}^{-1} \mu \mathrm{m}^{-1}$ for a wavelength of $10 \mu \mathrm{m}$, and a SNR threshold of 3 was applied. In this subsection we present a simple analysis that aims to quantify the possible influence of the noise level of the measuring device. For every differential signature in the spectral library we added a white noise that corresponds to SNR of 3, and calculated the spectral angle (SAM) between the original and noisy spectra. As detailed in Sect. 4, our analysis utilized a SAM threshold of $10^{\circ}$ during the validation experiment. Therefore, we consider a differential spectrum to be affected by noise only when the SAM angle between the original and noisy spectra exceeds $5^{\circ}$. Our analysis indicates that the proposed methodology is quite robust, as clouds with LWC higher than $13.8 \mathrm{mg} \mathrm{m}^{-3}$ are not affected by the random noise.

\subsubsection{Method's sensitivity to instrumental spectral features}

Every measuring device suffers from inherent bias. Spectroradiometer can suffer from different biases at different wavelength which might be interpreted as inherent spectral features, and in this subsection we have analyzed the effect of such features in terms of possible misclassification of the proposed methodology. At first, a long time series of differential spectral signals of a blackbody was measured in the laboratory, using the same measurement parameters (FOV, acquisition rate) as in the field campaign. Then, the commonly used technique of PCA - principal component analysis was applied (Johnson and Wichern, 1992). PCA considers the data as a matrix which is composed of $\boldsymbol{p}$ vectors, which stands for the $p$ variables in the data (wavelengths in our spectral analysis). Algebraically, principal components are particular linear combinations of the $\mathrm{p}$ random variables. Geometrically, these linear combinations represent the selection of a new coordinate system. The axes in the new coordinate system represent the directions with maximum variability in the original dataset. Technically, PCA calculates the covariance matrix of the dataset and finds its eigenvectors. Since the dataset was acquired by using a blackbody, every eigenvector represent an inherent spectral feature of the radiometer. Moreover, the eigenvalue of every eigenvector represents the amount of variance in the data which can be accounted for by the eigenvector. As stated previously, the proposed method utilizes 67 spectral bands, and therefore the PCA produced 67 eigenvectors. In order to examine whether the measuring device contains inherent spectral features that might induce bias to our methodology, we used the same analysis which is detailed previously to compare the spectral similarity between these eigenvectors and the expected spectra of thin clouds at the spectral library which was produced by MODTRAN. The spectral angle (SAM)

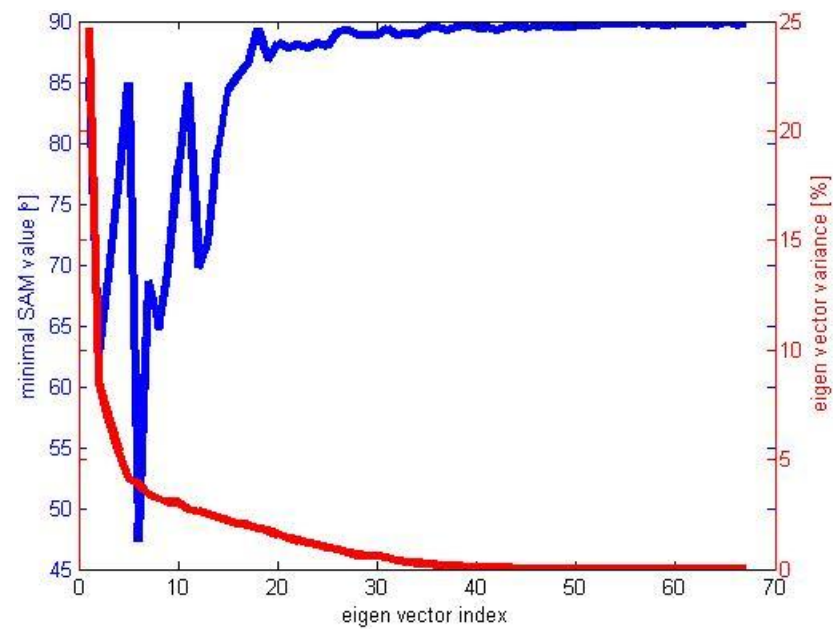

Fig. 11. The possible effect of inherent spectral noise of the measuring device on the proposed methodology. The eigenvectors are sorted according to their variance (red line), as commonly presented in principle component analysis. The blue line is the smallest (spectrally closest) SAM value between every eigenvector and the clouds spectral library. These high SAM values indicate that the inherent features spectrally differ from expected clouds signals, and cannot induce any misclassification on the proposed methodology.

between every eigenvector and every cloud differential signal was calculated (Fig. 11). The red line in Fig. 11 is the total variance of every eigenvector (sorted in descending order as commonly presented in PCA analysis), and the blue line is the lowest (spectrally closest) SAM value between every corresponding eigenvector and the clouds spectral library. One can notice that the closest SAM value between any of the eigenvectors and the clouds signals stands on $46^{\circ}$, while the SAM threshold applied in our study is $10^{\circ}$. The analysis, along with the usage of a signal to noise ratio (SNR) threshold of 3 (in wavelength of $10 \mu \mathrm{m}$ ) on the measured signal, suggests that inherent noise and spectral features cannot affect our methodology.

\subsubsection{Method's sensitivity to the number of spectral bands}

As mentioned above, the SAM analysis utilized 67 spectral bands that are measured by the spectro-radiometer between $8-13 \mu \mathrm{m}$. Due to practical and technical reasons, most developers of remote-sensing techniques prefer to use as low as possible number of spectral bands in their analysis. In light of the above, a simple analysis was conducted to check whether the spectral variability holds when the number of spectral bands is reduced. Since it is practically impossible to check all the permutations of the original spectral bands, a simple bands reduction iterative scheme was applied: in a specific iteration, where $n$ wavelengths remained, $n$ possible cross SAM matrices were calculated. Every cross SAM matrix was calculated by eliminating a different wavelength. 

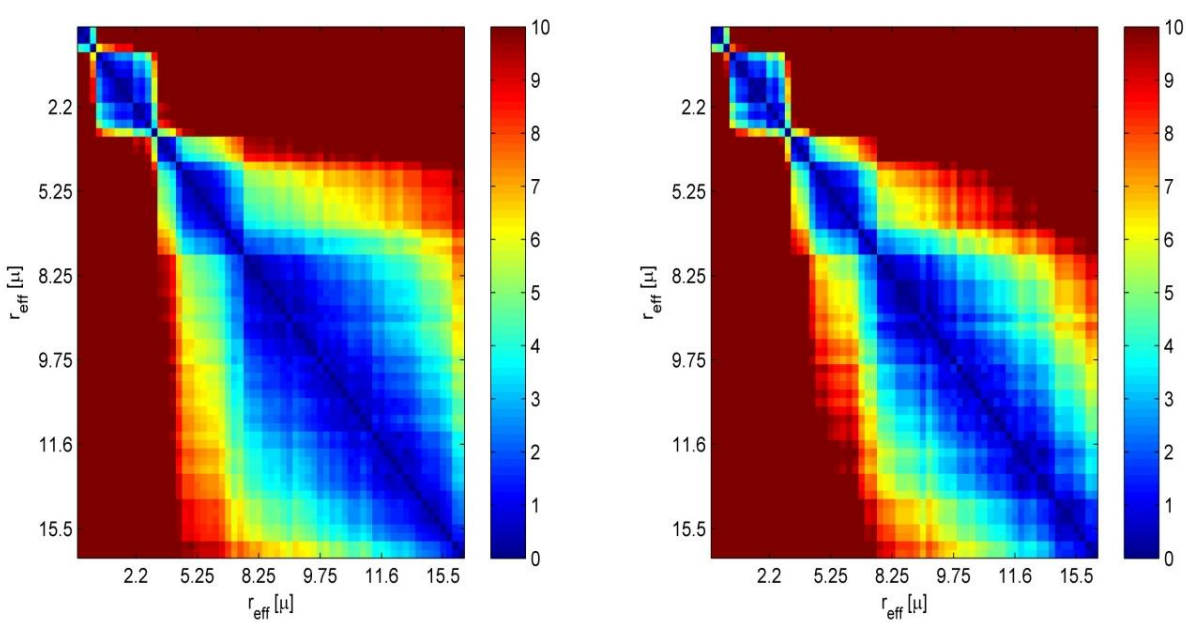

Fig. 12. The cross SAM matrix of differential spectral signals (of thin water clouds), using 67 spectral bands (left panel) and 5 spectral bands (right panel). One can notice the cross SAM matrix look similar which suggests that reduced number of bands can be used for the retrieval.

The best cross SAM matrix was found, and its corresponding wavelength was chosen to be eliminated. Where there might be several ways to define what is the best cross SAM matrix, we compared different matrices by the sum of their first diagonal. Large values indicate that the signatures largely differ from one another, whereas small values indicate the signatures are more spectrally similar. Our iterative procedure was applied until the number of bands was 5 .

Figure 12 compares the cross SAM matrix using only 5 spectral bands to the original cross SAM matrix (67 bands). It clearly shows that the spectral variability still holds even when 5 bands are used. In spite these encouraging results, it should be noted that a comprehensive analysis regarding the optimal number of bands should still be performed. Such analysis must consider more aspects of bands reduction, namely noise effect, misclassification, and possible biases.

\subsection{Induced bias or misclassification as a result of fluctuations in the relative humidity, aerosols, and haze}

The purpose of the proposed methodology is to extract the properties of water clouds with very small optical depth, by analyzing the spectrum and the magnitude of the signal. Fluctuations in relative humidity, aerosol loading, and haze are also characterized by small optical depths. Since the proposed method is based on subtraction of clear and cloudy zenith sky spectrum, it is essential to examine whether such fluctuations and thin water clouds can alter the sky radiance in a similar way (in terms of spectrum and magnitude). If such similarities exist, the proposed method might falsely interpret such fluctuations as thin water clouds. In addition, when thin water clouds do exist in the sensor's FOV, fluctuations in water vapours and aerosols might induce a bias to the retrieved parameters.
In this subsection we will analyze the zenith sky radiance in the presence of fluctuations in relative humidity, aerosols, and haze. The spectral change as well as the magnitude of the signal will be analyzed, and we will estimate or try to bound the bias that might be induced to the retrieved parameters as a result of such fluctuations.

\subsubsection{Relative humidity}

Water vapour molecules are a substantial constituent of the troposphere and are one of the key parameters which determine the measured thermal radiation of the sky. Although water vapours does not scatter the LWIR radiation, its absorption alters the downwelling sky radiance. Therefore it is essential to verify that short term variations in the water vapour column distribution do not induce substantial changes in the sky radiance, compared to the changes induced by thin water clouds. At first, we used radiative transfer calculations to analyze the magnitude of the expected change in the sky radiance as a result of fluctuations in water vapour. For the mentioned atmospheric profile, our modelling results predict that the expected change in the sky radiance at wavelength $10 \mu \mathrm{m}$ is $1.52 \times 10^{-5}$ Watts $\mathrm{cm}^{-2} \mathrm{str}^{-1} \mu \mathrm{m}^{-1}$ when the relative humidity in the layer of $800-850 \mathrm{~m}$ raises from $10 \%$ to $100 \%$. This radiative difference is small since the total transmittance of a $50 \mathrm{~m}$ layer of water vapours at temperature of $26.23{ }^{\circ} \mathrm{C}$ (the air temperature of the layer in the chosen atmospheric profile) is 0.992 (therefore the layer emissivity is 0.008). It suggests that even such extreme and unlikely fluctuations are expected to create a change in the measured sky radiance smaller than our predefined SNR threshold $\left(1.92 \times 10^{-5}\right.$ Watts cm ${ }^{-2} \mathrm{str}^{-1} \mu \mathrm{m}^{-1}$, corresponding to 3 times the NESR of the SR5000), and therefore fluctuations in water vapour cannot be falsely interpreted as thin water clouds. 


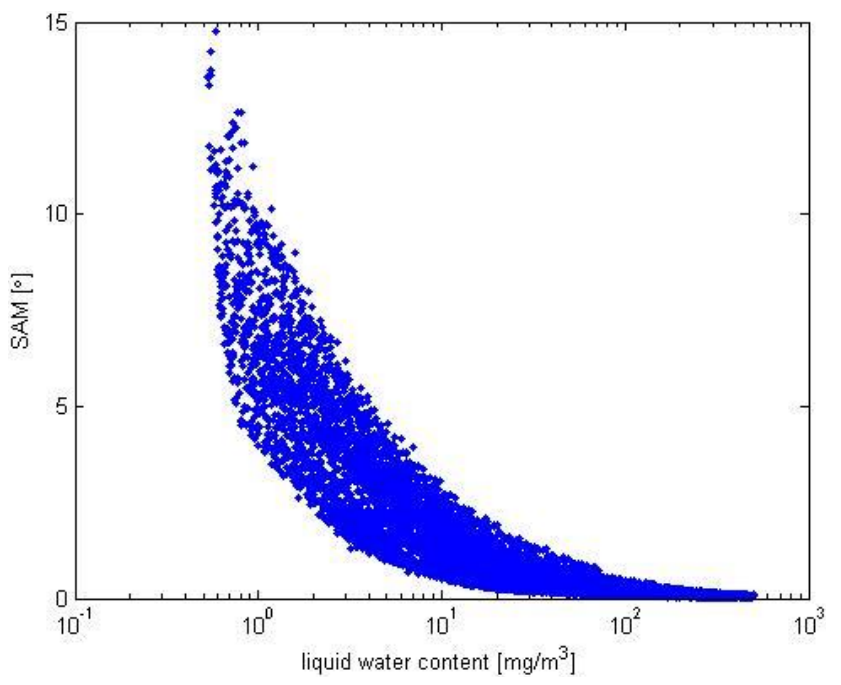

Fig. 13. SAM between every simulated cloud's differential spectral signature and the modified differential spectral signature which is the result of a 50 meter thick layer $(750-800 \mathrm{~m})$ below the cloud, with the relative humidity as set to $50 \%$. Note that this scatter plot contains information regarding all the simulated clouds, with varying geometrical depths (as described in Table 2), and that the horizontal scale is the LWC of the simulated clouds. It is noticeable that only clouds with LWC less than $4 \mathrm{mg} \mathrm{m}^{-3}$ might experience a spectral shift of more than $5^{\circ}$ in the SAM index.

In addition, the possible bias due to water vapour column fluctuations over the retrieved parameters of thin clouds in the sensor's FOV was analyzed. In order to examine this possible effect quantitatively, we used radiative transfer calculations to predict the sky spectrum in the presence of thin clouds with two different humidity profiles. The first profile was the actual sounded atmospheric profile which was measured by the radiosonde and indicated a relative humidity of $32 \%$ in the $50 \mathrm{~m}$ layer below the cloud base $(750-800 \mathrm{~m})$. The second profile was the same as the first one, except the relative humidity in the layer below the clouds, which was modified to a value of $50 \%$. The spectral angle (in terms of SAM) between each pair of differential sky spectra was calculated, as presented in Fig. 13. As seen in the figure, only clouds which contained LWC lower than $4 \mathrm{mg} \mathrm{m}^{-3}$ might pose a spectral shift higher than $5^{\circ}$. We repeated this analysis by altering the relative humidity (in the 750-800 m layer) in the range of 10-100\%. The assessment of the relative humidity impact is shown in Fig. 14. For every value of the relative humidity, we analyzed the maximal LWC that will induce a spectral shift higher than $5^{\circ}$. The main conclusion from this analysis is that even extreme and unlikely fluctuations in the relative humidity layer below the cloud, might affect clouds with LWC of no more than $35 \mathrm{mg} \mathrm{m}^{-3}$.

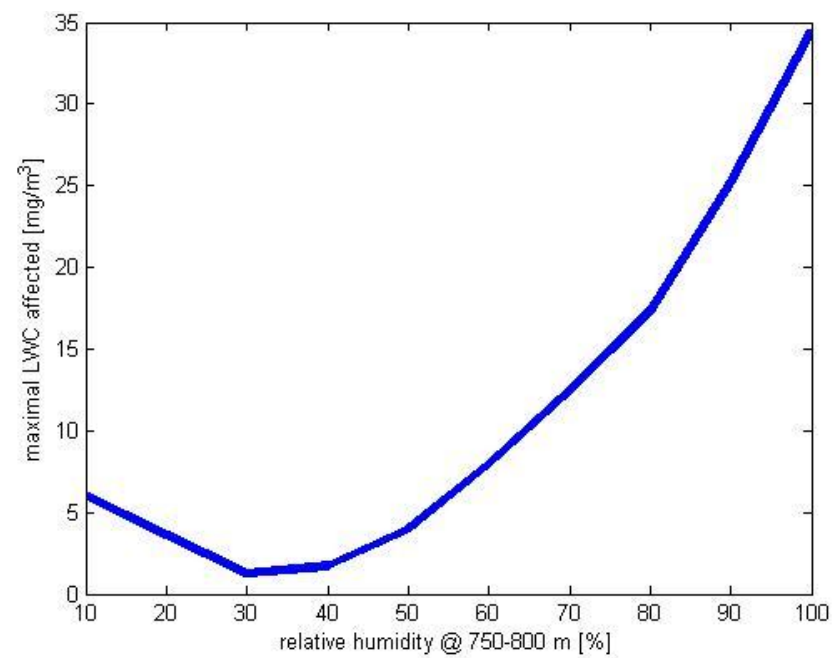

Fig. 14. Clouds with LWC lower than the values shown in the graph will be affected by variations in the relative humidity values of the layer below the clouds $(750-800 \mathrm{~m})$. A cloud is considered to be affected if its differential spectral signature differs by more the $5^{\circ}$ (in terms of SAM) from the differential spectral signature of the modified cloud.

\subsubsection{Aerosols}

The origin of aerosols in the atmosphere is attributed to many sources and can be emitted by either natural processes or by anthropogenic activity (IPCC, 2007). The vast majority of these airborne particles are small compared to water droplets, and as a result their radiative effect is mostly noticeable in the visible portion of the spectrum. Nevertheless, measurements in the IR regime have been occasionally made. Aerosols' IR optical properties during the ACE-Asia campaign were measured (Markowicz et al., 2003), and it was shown that the mean aerosol optical depth at $10 \mu \mathrm{m}$ was 0.08 (for the entire atmospheric column). Apart from the complete optical depth, aerosol optical properties in the LWIR have been studied (Thomas et al., 2005; Richwine et al., 1995; Toon et al., 1976; Volz, 1972, 1973), and in addition, the effect of changes in the relative humidity on the extinction coefficients of atmospheric aerosols was presented (Nilsson, 1979). These properties can be incorporated in radiative transfer models in order to determine aerosols' radiative effect. MODTRAN offers several predefined aerosol models, and in this study we have analyzed the effect of the rural, maritime, and urban models which will be described briefly in the coming paragraph. The rural model represents the aerosol conditions in continental areas which are not directly influenced by urban and industrial aerosol sources. The rural aerosols are assumed to be composed of a mixture of $70 \%$ of water-soluble substances and $30 \%$ dust-like aerosols. The size distribution of this aerosol model is parameterized as the sum of two log-normal size distributions, to represent the multimodal nature of the atmospheric aerosols. In addition, the aerosol size 

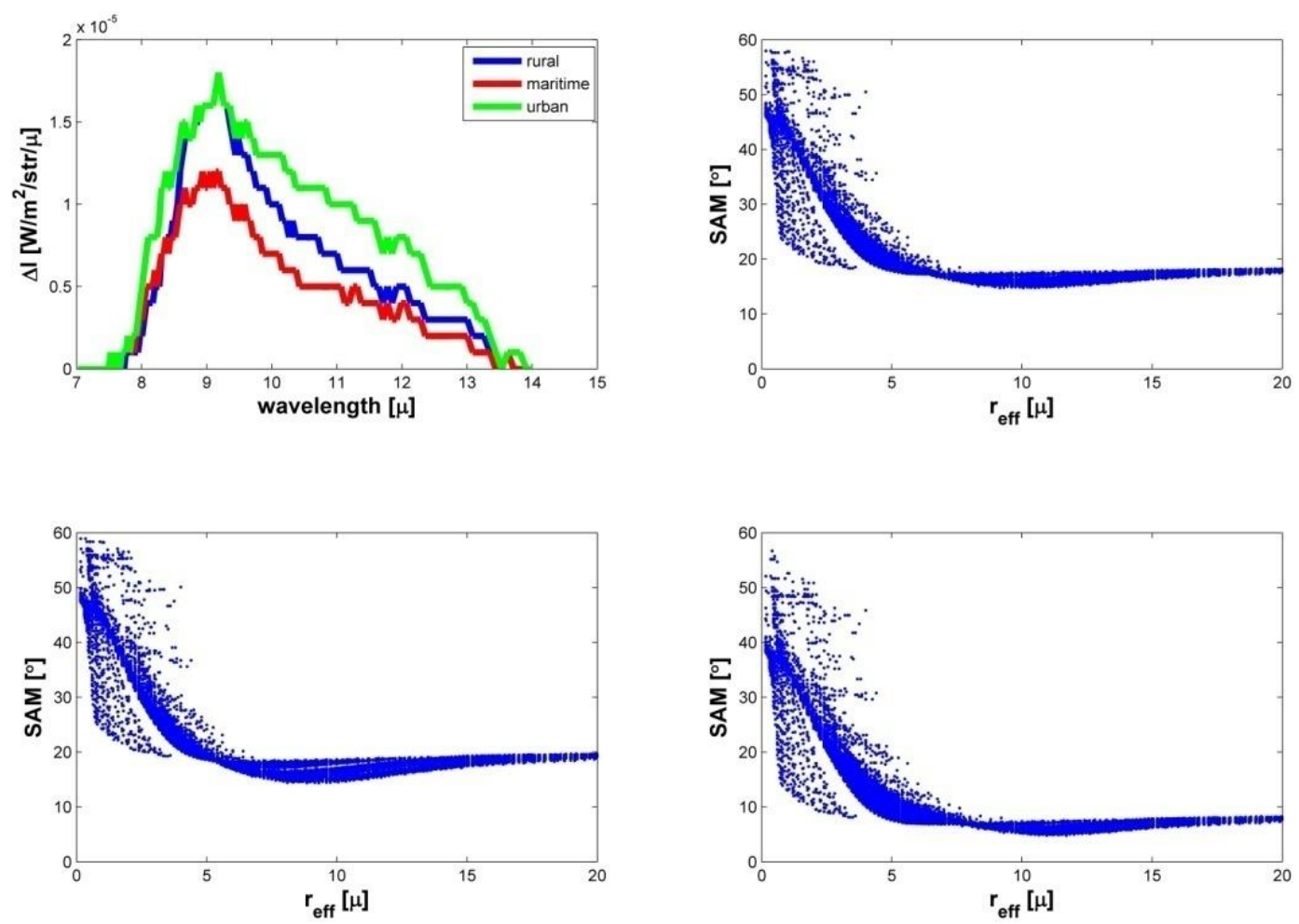

Fig. 15. Top left panel: the effect of atmospheric aerosols on the zenith sky spectrum. The differential spectrum of the zenith sky in the presence of an optically thick (AOT $=0.26$ ), $50 \mathrm{~m}$ aerosol layer at $800-850 \mathrm{~m}$. Top right panel: scatter plot of the SAM angle between the differential spectrum of rural aerosols (blue line in top left panel) and the differential spectra of thin water clouds with a certain effective radius (x-axis). Bottom left panel: the same, but for maritime aerosols. Bottom right panel: the same, but for urban aerosols. It seems that rural and maritime aerosols do not show any spectral similarity to water clouds, as the lowest SAM values are $14.76^{\circ}$ and $14.35^{\circ}$, respectively. However, since the minimal SAM value between urban aerosol spectrum and water clouds spectra is $4.79^{\circ}$, the urban aerosols effect might appear similar to water clouds to some extent. However, the magnitude of the expected change is relatively small, even in the extreme simulated theoretical conditions.

is determined as a function of the humidity in the layer in which they are simulated. The urban aerosol model is a modification of the rural model by an addition of aerosols from combustion products and industrial sources. The mixture is composed of $80 \%$ rural aerosols and $20 \%$ sootlike aerosols. The maritime aerosol model largely differs from the previous models, and is composed of two components: aerosols developed from sea spray, and a continental component assumed identical to the rural aerosol with the exception that the very large particles were eliminated, since the will eventually be lost due to fallout. These aerosol models are characterized by their optical properties in the visible and the LWIR region of the spectrum, and can be readily incorporated in MODTRAN in a certain atmospheric layer by specifying the aerosol's extinction coefficient for unit length at a wavelength of $550 \mathrm{~nm}$.

To estimate the effect of aerosols on the retrieved methodology we used the mean aerosols optical thickness (AOT) that was measured in a local AERONET site (Holben, et al., 1998, http://aeronet.gsfc.nasa.gov/). AERONET's average AOT during the months of August through October 2010 was 0.26 at $500 \mathrm{~nm}$. We have assumed an extreme scenario where all the atmospheric aerosols are concentrated in a $50 \mathrm{~m}$ layer, thus MODTRAN's required input for extinction coefficient was calculated to be $\sigma(\lambda=550 \mathrm{~nm})=0.26 / 0.05=5.2 \mathrm{~km}^{-1}$. It is important to note that such extreme extinction causes the optical visibility (defined as $2 \%$ at wavelength of $550 \mathrm{~nm}$ ) to be $752 \mathrm{~m}$, and it is typical for heavy dust storms (during which, all the lower troposphere is filled with dust). Obviously, this assumption enhances the radiative effect of aerosols on the sky spectrum. The top left panel in Fig. 15 presents the expected differential spectrum in the presence of such aerosols in the atmospheric layer between $800 \mathrm{~m}$ to $850 \mathrm{~m}$, using the atmospheric profile of 8 August 2010. One should compare the magnitude of the differential signals which are expected by a thin water cloud with LWC of $15 \mathrm{mg} \mathrm{m}^{-3}$ (Fig. 5) and thick, dust-storm like aerosol layer (top left panel in Fig. 15). It is noticeable that even extreme, unrealistic aerosol condition which were simulated, are expected to produce a signal which is still an order of magnitude less than is expected for a thin water cloud.

To estimate the spectral effect of aerosols on the retrieved parameters, we calculated the SAM index between 
every aerosol model's differential spectrum and the simulated clouds' differential spectrum. The SAM angle values for the different aerosol models are presented in Fig. 15. It is notable that rural and maritime aerosols do not pose any significant spectral bias as the minimal (spectrally close) SAM angle between the aerosols and the clouds stands at $14.76^{\circ}$ and $14.35^{\circ}$ respectively. However, it seems that the presence of urban aerosols can affect the sky radiance in a way which is similar to the effect of very thin clouds with $r_{\text {eff }}>2 \mu \mathrm{m}$, since the minimal SAM angle between the urban aerosols and the clouds is $4.79^{\circ}$. Nevertheless, even in a scenario where all the aerosols are concentrated in a $50 \mathrm{~m}$ layer, and the optical properties of the layer resemble that of a dust storm, the magnitude of the differential spectrum caused by the urban aerosols is very low: only a $6 \%$ increase in the radiance at wavelength of $10 \mu \mathrm{m}$ compared to the clear sky signal. In any case, as described in Sect. 4.1, this increase represents a signal to noise ratio of 2 (compared to the measuring device) and we have discarded any signals with SNR lower than 3. This analysis shows that the influence of tropospheric aerosols is marginal for any practical purpose, and that their effect on the retrieved cloud parameters is negligible.

\subsubsection{Haze}

The growth of a water droplet due to condensation is well described by the Köhler (1936) curve. The required ambient supersaturation needed to support spontaneous condensational growth of the droplet depends on the exact chemical composition of the solution droplet as well as on the droplet's radius. When the ambient supersaturation is high enough, the droplet can pass a thermodynamic barrier represented by the Köhler peak. Such a droplet is said to be activated and will continue to grow by condensation and become a cloud droplet. On the other hand, insufficient ambient supersaturation will cause a droplet to stay in stable equilibrium. Under these conditions, a slight increase in its size (by condensation) will cause immediate evaporation that will bring it back to the equilibrium size, and a slight decrease in its size (by evaporation) will result in condensation that will return its size. Such unactivated droplets are called haze droplets.

The proposed methodology presented in this study can distinguish between water droplets with different radii due to their optical properties, but it obviously cannot separate between different microphysical states. Nevertheless, the typical effective radius of haze cloud is $0.05 \mu \mathrm{m}$ (Deirmendjian, 1964), yielding a volume extinction coefficient in the IR band, which is about 3 orders of magnitude smaller than that of a typical water cloud. These kinds of haze clouds are expected to have a negligible effect on the obtained effective radius and optical depth of thin clouds. Therefore, we are quite confident to conclude that when the effective radius of a certain cloud is retrieved, it represents an activated water cloud rather than a haze cloud.

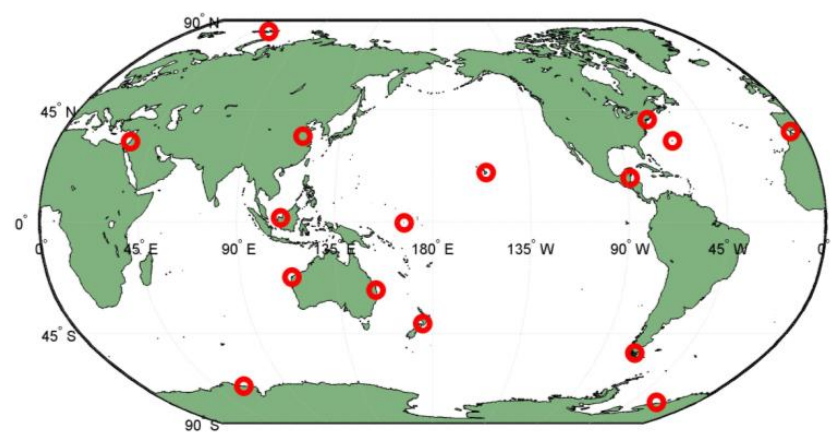

Fig. 16. The location of 16 worldwide meteorological stations (http: //weather.uwyo.edu/upperair/sounding.html), which were manually chosen. The atmospheric profile was measured simultaneously on 8 August 2010, 12:00 UTC. These locations represent wide range of seasonal, diurnal, and meteorological conditions (from Arctic across the tropics to Antarctica, and both summer and winter).

\subsection{Sensitivity of the proposed method to variations in the atmospheric profile}

The analysis presented in the former sections utilized a certain atmospheric profile which was measured in a nearby meteorological station on a specific day. In order to study the efficacy and applicability of the proposed method it is important to analyze its sensitivity to the temperature and humidity profile. Therefore, we picked 16 atmospheric profiles (http://weather.uwyo.edu/upperair/sounding. $\mathrm{html}$ ), which were measured simultaneously at different locations around the globe (Fig. 16), and repeated the analysis presented in Sect. 3.1 to create a spectral library of thin clouds for every measured profile.

Figure 17 presents the expected clear sky zenith radiance under the different atmospheric profiles. As expected, the radiance of the zenith sky is smaller under drier and colder atmospheric conditions, and noticeable variations exist in the clear sky spectrum, both in terms of the spectral shape and in the magnitude of the signal. As detailed in Sect. 2, the methodology relies on spectral analysis of the variability of the differential spectrum which is created by subtracting the clear sky signal from the zenith sky radiance in the presence of a thin cloud. Figure 18 presents the expected differential spectrum under the different atmospheric profiles in the presence of a thin warm cloud characterized by an effective radius of $2.1 \mu \mathrm{m}$ and LWP of $750 \mathrm{mg} \mathrm{m}^{-2}$. It is noticeable that the magnitude of the differential signal is comparable under all atmospheric profiles which suggests that in terms of signal to noise ratio, the proposed method can be used globally.

Nevertheless, examining Fig. 18 reveals spectral variability that exists for the same cloud under different atmospheric profiles. Quantifying this spectral variability in terms of SAM (Fig. 19) provides interesting insights on the applicability of the proposed method: clouds under similar atmospheric conditions will have small cross SAM values 


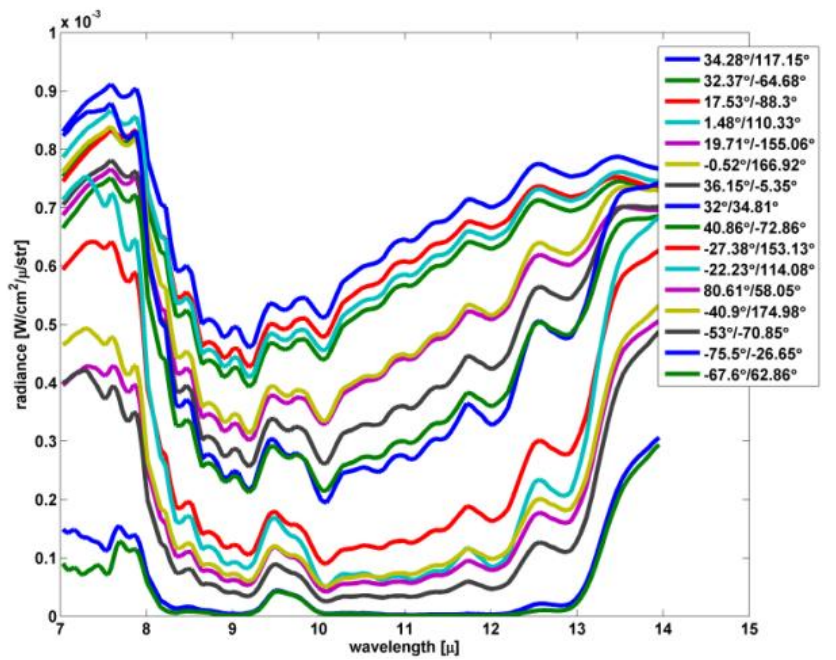

Fig. 17. The expected spectral radiance of the zenith clear sky under different atmospheric profiles.

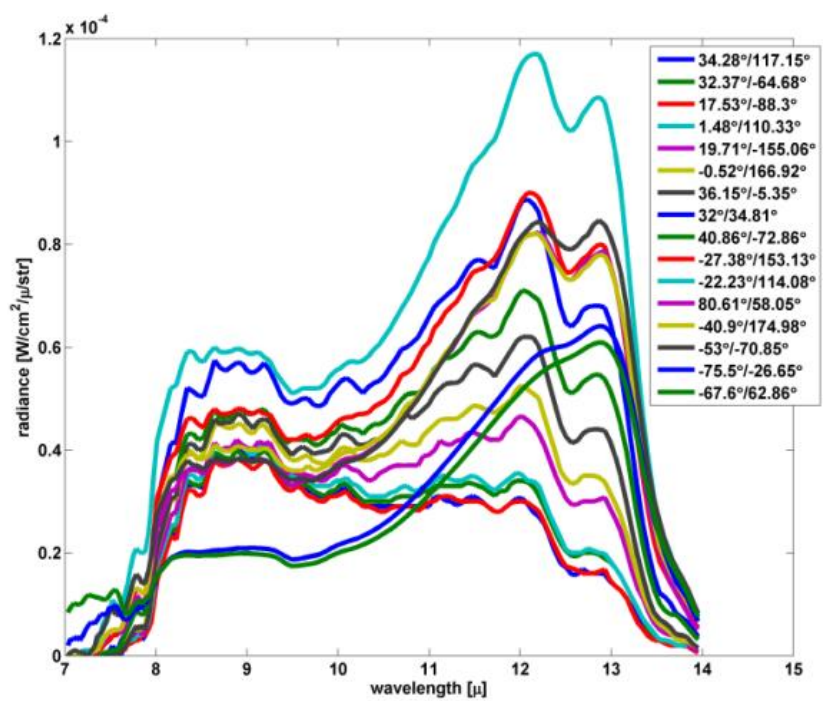

Fig. 18. The expected differential spectrum of a thin cloud under different atmospheric profiles. The cloud is characterized by and effective radius of $2.1 \mu \mathrm{m}$ and LWP of $750 \mathrm{mg} \mathrm{m}^{-2}$.

(i.e. their spectral signatures will resemble each other), whereas clouds under extremely different atmospheric profiles can differ from each other by a cross SAM value of up to $\sim 30^{\circ}$.

The analysis presented in this subsection suggests that the proposed methodology is applicable for global use under wide range of seasonal, diurnal, and meteorological conditions. However, the analysis emphasizes that adequate characterization of the observing system is necessary in order to retrieve valid and trustable results, and one must rely on measured representative atmospheric profiles (in terms of general location and season) and a trustworthy radiative transfer model that can incorporate user-defined profiles.

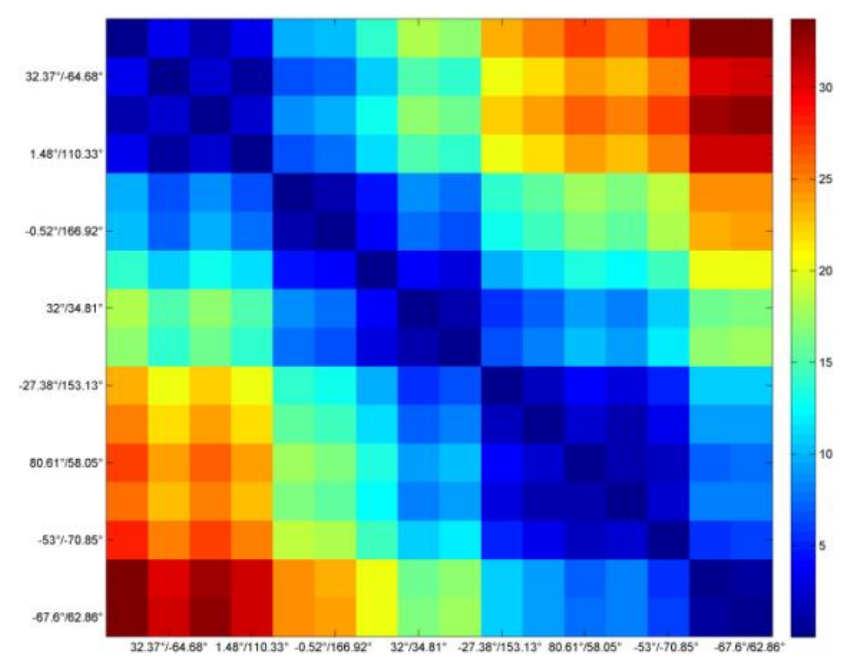

Fig. 19. Cross SAM values of differential spectrum of the same cloud under different atmospheric profiles. The axes labels indicate the latitude/longitude of the different meteorological stations.

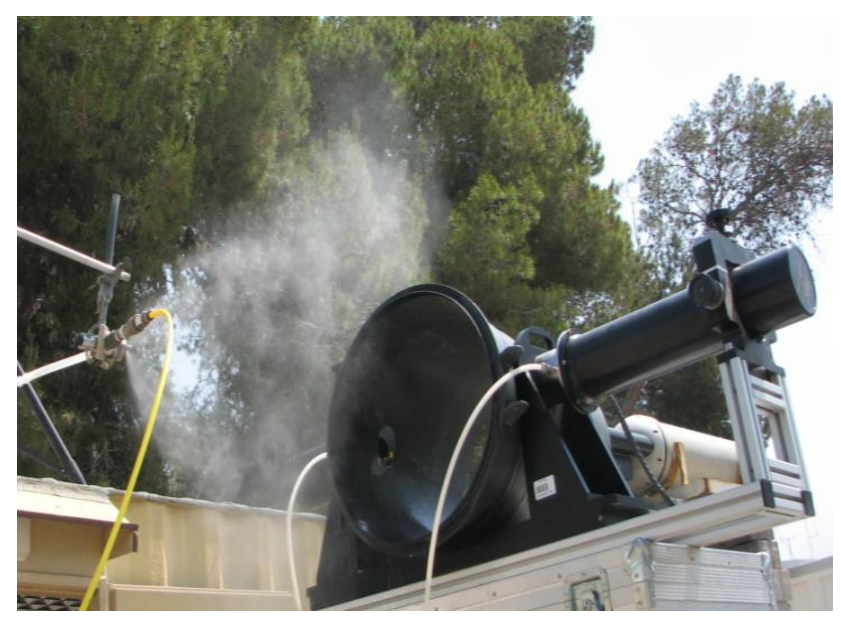

Fig. 20. A controlled experimental setup for validation of the proposed methodology. An artificial cloud was sprayed via an airatomizing nozzle and entered the field of view of an accurate particle sizing device (Spraytec). A calibrated spectro-radiometer and a thermal imager were positioned below the passing cloud (not shown), pointing to the zenith.

\section{Validation of the proposed methodology in a controlled environment experiment}

In order to validate the proposed methodology, a controlled experiment was conducted outdoors (Fig. 20). The purpose of this small-scale experiment was to validate the main concept that constructs the basis of the proposed methodology. Mainly, the usage of a radiative transfer model combined with spectral analysis allows for the retrieval of cloud water droplet effective radius (assuming the type of the droplets size distribution is known). In addition, a comparison between the retrieved and measured LWC was conducted. An 


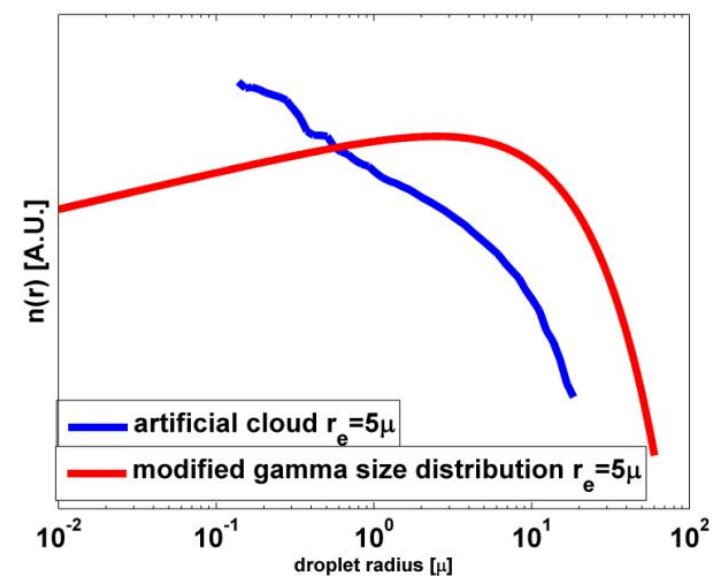

Fig. 21. Modified Gamma number size distribution (red), and the number size distribution produced by the air atomizing nozzle (blue). Both distributions are characterized by effective radius of $5 \mu \mathrm{m}$.

artificial water cloud was sprayed with an air-atomizing nozzle, and the cloud's droplets size distribution was varied by altering the air pressure supplied to the nozzle. Our main instrumental device was the SR5000 (see Appendix A for further details). In addition, a thermal imager (A40, FLIR SYSTEMS, USA) was used in order to continuously acquire images of the zenith sky and artificial cloud. This broadband $(7-14 \mu \mathrm{m})$ IR imager is based on an uncooled microbolometer sensor with $240 \times 320$ pixels, calibrated to effective radiant temperature. It has a wide FOV $\left(18^{\circ} \times 24^{\circ}\right.$, equivalent to $314 \mathrm{mradX} 419 \mathrm{mrad}$ ) and its acquisition rate was set to $6.25 \mathrm{~Hz}$. Complementary to the on-site measurement assembly, we used the atmospheric profile which was measured in the meteorological station in Beit-Dagan (http:// weather.uwyo.edu/upperair/sounding.html), located approximately $10 \mathrm{~km}$ from our measurement site. This atmospheric profile was used as an input for the radiative transfer calculations, as explained in the previous sections. Immediately after the cloud passed over our sensors, it entered the field of view of an accurate particle sizing device (Spraytec, Malvern, UK), which continuously measured the normalized volume size distribution of the droplets.

As stated before, the purpose of the controlled experiment was to validate the proposed methodology. Unfortunately, the air-atomizing nozzle cannot produce a droplet size distribution which follows the Modified Gamma size distribution (Eq. 2). The differences between these size distributions are revealed in Fig. 21. Whereas the Modified Gamma size distribution is characterized by a distinct peak and absence of droplets below a certain threshold, the nozzle produces number concentration which is almost monotonic decreasing. Since one of the basic concepts in our method is spectral match between the measured differential signal and a spectral library created by a radiative transfer model, and since

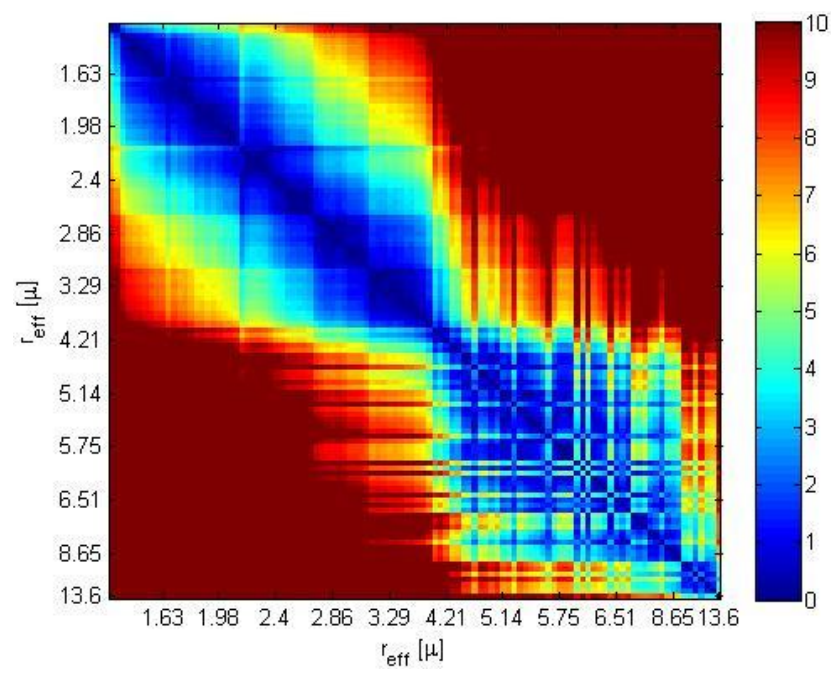

Fig. 22. Cross SAM matrix of differential signals of thin water cloud (LWC $\sim 15 \mathrm{mg} \mathrm{m}^{-3}$ ) that follow the droplets size distribution that is produced by the air-atomizing nozzle.

the predicted signal strongly depends on the droplets size distribution, it is essential that the spectral library be based on correct information regarding the droplets size distribution. Therefore, we have used droplets size distribution readings of the Spraytec during the experiment as an input for MODTRAN to create the spectral library. Figure 22 presents the cross SAM matrix of the differential signals which were created for the validation experiment. Comparing Figs. 22 and 8 reveals the differences between the validation experiment and the theoretical capabilities of the method regarding natural clouds. Two main distinct areas exist in the cross SAM matrix in Fig. 22: spray with effective radius of less than $4 \mu \mathrm{m}$ differs from spray with higher effective radius. The ability to distinguish between sprays within the two areas is quite small.

After the proper spectral library was created, we have utilized the proposed methodology. As described in Sect. 2.4, the rationale behind the retrieval methodology is to choose the possible solutions that match our observation and produce the best results in terms of both spectral fitness and signal magnitude. Specifically, the following scheme was used to determine the valid solution space for every measured spectrum:

a. Let $I$ be a spectral signature of the zenith sky at some point in the analyzed time frame, and let $S$ be the spectral library of the differential spectral signatures of the simulated clouds.

b. Let $I_{\text {ref }}$ represent the clear sky spectrum (measured by the SR5000 just before the artificial cloud was sprayed).

c. We define the measured differential spectral signature to be $\Delta I=I-I_{\text {ref }}$. 


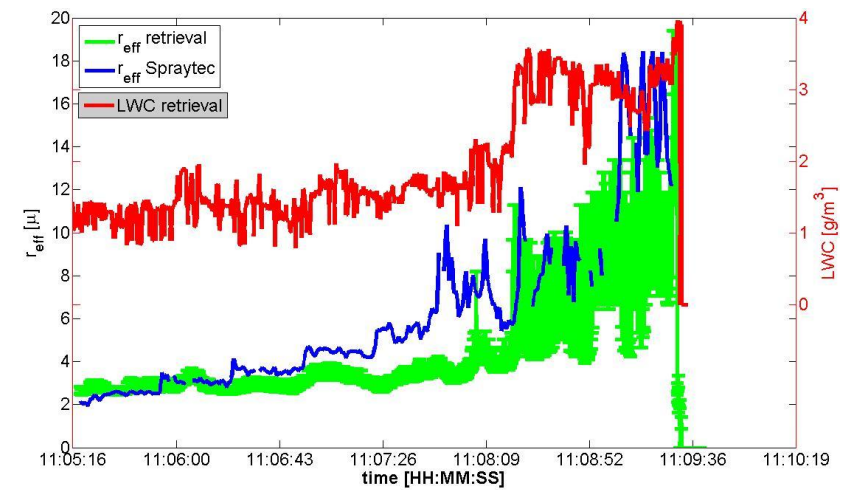

Fig. 23. The result of the validation experiment: retrieved LWC and effective radius of the artificial cloud which was sprayed during the controlled validation experiment. Blue: effective radius measured by the Spraytec. Green: effective radius retrieved by the proposed methodology. Red: the retrieved LWC of the cloud, as produced by the algorithm. (The geometrical thickness of the cloud is implemented in the retrieval, see Sect. 2.4.) The range of the retrieved effective radius is the result of allowing 10 valid solutions (see stage "G" in Sect. 4).

d. Let $S_{A M} M_{\text {RES }}$ be a vector containing the calculated SAM values between $\Delta I$ and every signature in $S$.

e. Let $S_{\text {sub }}$ be a subset of S which contains all the differential spectral signatures of the simulated clouds, for which the SAM RES is smaller than $10^{\circ}$.

f. Let $\mathrm{RMS}_{\text {sub }}$ be a vector of the root-mean-square (RMS) value of the spectral difference between every signature in $S_{\text {sub }}$ and $\Delta I$.

g. The simulated cloud with the lowest RMS value in $\mathrm{RMS}_{\text {sub }}$ corresponds to the best solution of the retrieval method. In order to allow a certain space of valid solutions, we consider the 10 simulated clouds which produced the lowest RMS values in the RMS $\mathrm{Sub}_{\text {sub }}$ vector to be the valid solutions for the measured signal $I$.

Figure 23 presents the results of the validation experiment. The blue and green lines are the effective radius of the artificial cloud as measured by the Spraytec, and retrieved by the proposed method, respectively. The red line is the retrieved LWC of the artificial cloud. One can notice that the retrieval largely agrees with the Spraytec's readings: if we define agreement between the Spraytec's and the retrieval's effective radii as a case where the Spraytec's effective radius does not deviate by more than $30 \%$ of the retrieval effective radius range, then the Spraytec and the retrieval agree more than $70 \%$ of the time. Some of the disagreements between the graphs are likely the result of the inevitable differences between the line of sights of the two optical devices: the Spraytec was positioned horizontally in a manner that assured most of the cloud entered its field of view all of the time. The SR5000, on the contrary, was pointed to the zenith

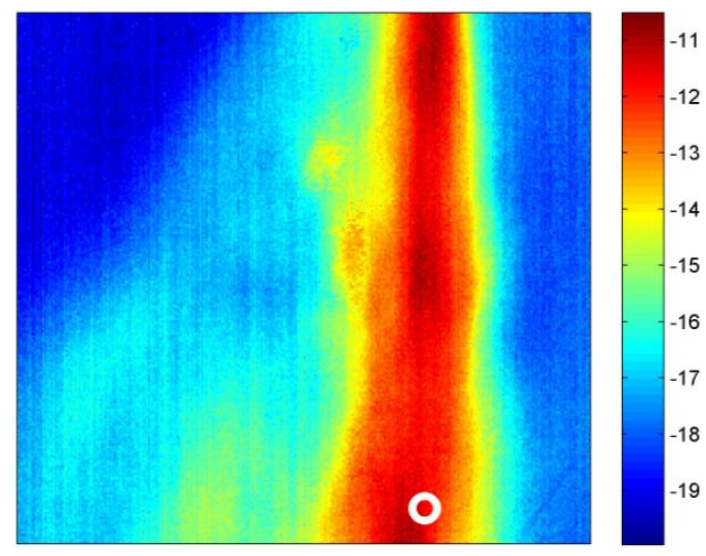

Fig. 24. A thermal image of the zenith sky (units in ${ }^{\circ} \mathrm{C}$ ) during the validation experiment. The passing artificial cloud emerges from the nozzle which is located at the upper side of the image (not shown), and enters the field of view of the Spraytec, which is located at the lower side of the image (not shown). As expected, the passing cloud appears warm over the cold sky background. The white circle is the estimated position of the SR5000's field of view. Since the artificial cloud is relatively narrow, the apparent cloud in the SR5000's field of view is highly sensitive to fluctuations in the wind direction.

with a mere field of view of $6 \mathrm{mrad}$ which corresponds to approximately $100 \mathrm{~mm}^{2}$ of the passing cloud. This setup made the retrieval of the SR5000 much more sensitive to wind fluctuations (Fig. 24).

Regarding the retrieved LWC (red line in Fig. 23), the performance is further encouraging. The retrieved LWC varies in the range of $0.78-3.97 \mathrm{~g} \mathrm{~m}^{-3}$ while the average retrieved LWC is $1.92 \mathrm{~g} \mathrm{~m}^{-3}$. Due to technical difficulties, a continuous measurement of the LWC was not possible. However, a reasonable estimation was possible by measuring the flow rate of the water from the air nozzle $\left(3 \mathrm{~g} \mathrm{~s}^{-1}\right)$, and analysis of the spray's speed $\left(4 \mathrm{~m} \mathrm{~s}^{-1}\right)$ and dimensions $\left(0.28 \mathrm{~m}^{2}\right)$ by using the FLIR images. Such experimental setup is expected to create a cloud with LWC of $2.68 \mathrm{~g} \mathrm{~m}^{-3}(=3 / 4 / 0.28)$.

\section{A case study}

A field campaign was conducted during the summers of 2010 and 2011, and the optical and microphysical properties of thin clouds were retrieved by the proposed method. A comprehensive analysis regarding the frequency and properties of such thin clouds will be published in a following paper. However, a case study of a single measurement is provided in order to demonstrate the method's capabilities. Figure 25 presents different stages through the retrieval process of data which was acquired on 15:43 LT (local time) 29 June 2011. A small, thin cloud passed over our sensors (upper panel in Fig. 25), while the SR5000 measured the sky zenith spectrum (bottom left panel). After calculating the differential spectra (black line in bottom right panel), the algorithm found the 

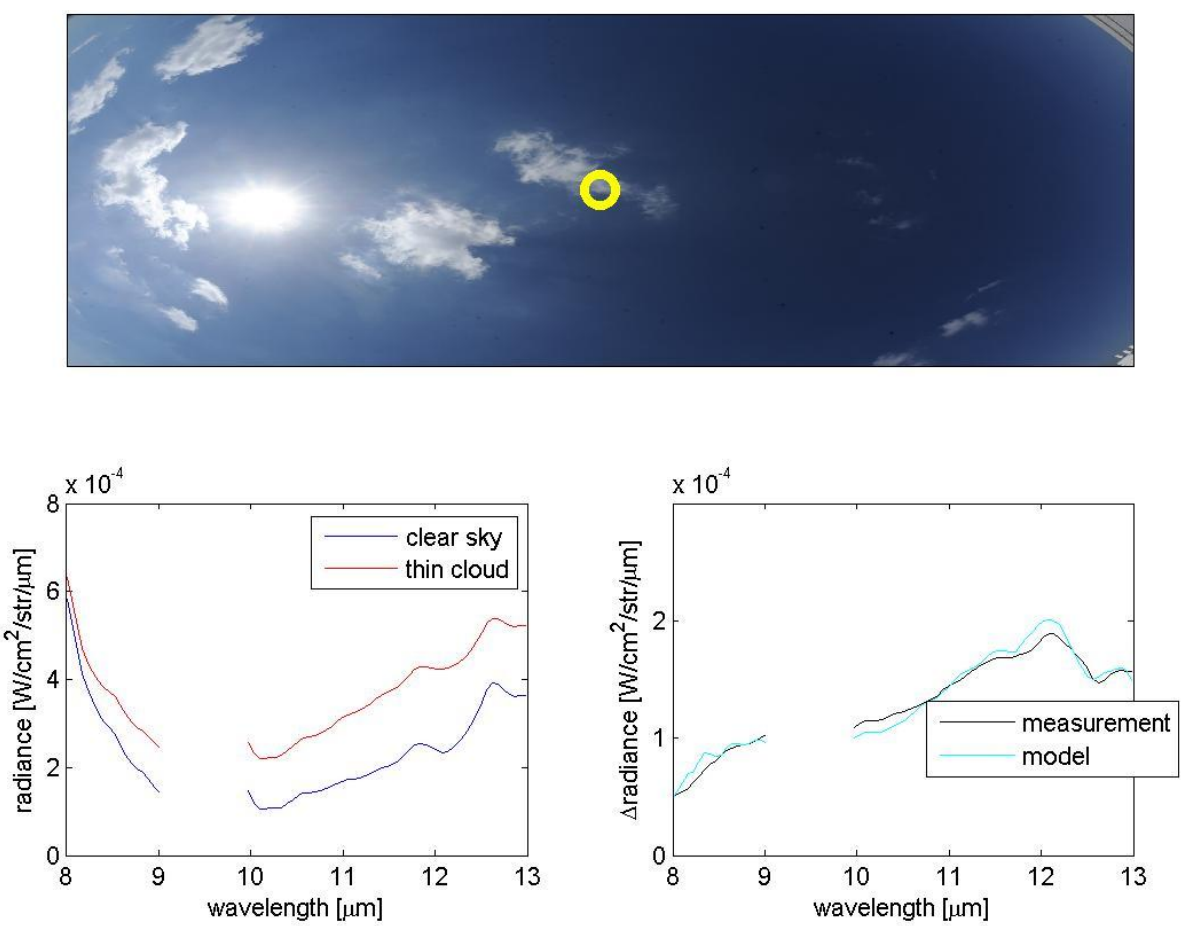

Fig. 25. A case study of a small, thin cloud which passed over our sensors at 15:43 LT (local time) 29 June 2011. Upper panel: a wide-angle (FOV of $180^{\circ}$ on the diagonal) RGB image of the sky hemisphere. The yellow circle indicates the estimated field of view of the SR5000. Bottom left panel: the sky zenith spectrum when the cloud was present in the sensor's FOV (red line), and the clear sky spectrum which was measured few minutes prior to the cloud's arrival (blue line). As explained, the $9-10 \mu \mathrm{m}$ region was omitted since it contains a relatively strong absorption band of $\mathrm{O}_{3}$. Bottom right panel: the calculated differential spectrum (black), and the best fit spectrum that was chosen by the algorithm from the spectral library (cyan). One can notice how the algorithm chooses a solution which fits the measurement in terms of spectral shape and magnitude of the signal. According to the proposed method the cloud was characterized by an effective radius of $1.35 \mu \mathrm{m}$, LWP of $1.713 \mathrm{~g} \mathrm{~m}^{-3}$, and optical depth of 1.9 (in the $550 \mathrm{~nm}$ wavelength).

best solution in terms of spectral shape and magnitude (cyan line in bottom right panel), following the same scheme that was described in Sect. 4. One can notice how the modelled and measured spectra almost perfectly match. The chosen solution represents a cloud with an effective radius of $1.35 \mu \mathrm{m}$, LWP of $1.713 \mathrm{~g} \mathrm{~m}^{-3}$, and optical depth of 1.9 (in the $550 \mathrm{~nm}$ wavelength).

\section{Conclusions and discussion}

A new concept for the retrieval of the microphysical and optical properties of very thin water clouds is proposed. The method utilizes ground based measurements which exploit high spectral, spatial, and temporal resolutions, and allow a study of cloud small scale dynamics and evolution, which cannot be measured in fine details from space, due to the sensor's insufficient temporal and spatial resolution. The retrieval methodology is composed of 3 elements: radiative transfer model, enhancement of the signal by subtraction of clear sky reference, and spectral matching method. The theoretical limitations of the methodology have been discussed, and the current estimate is a liquid water path range of $0.065-49.26 \mathrm{~g} \mathrm{~m}^{-2}$, and a lower limit of about 0.01 visible optical depth. The method is particularly efficient for clouds with effective radii of up to $4 \mu \mathrm{m}$. These thin clouds are practically ignored in common remote sensing measurements of cloud's properties as well as global circulation models, although their frequency (and their total radiative forcing) is expected to be high. The proposed method can be complementary to well established methods which were developed for thick clouds (see Yang et al., 2008; Chiu et al., 2010; McBride et al., 2011 for example), in order to derive a more profound analysis of the clouds properties. The proposed methodology was validated in a controlled experiment where artificial clouds were sprayed and their effective radii and LWC were measured and retrieved by the method simultaneously. We have also shown that changes in relative humidity, aerosols, and haze cannot be attributed as the source for the measured changes in the sky radiance, as their effects differ by either the expected spectral behaviour or the magnitude of the signal.

This methodology and ongoing measurements might prove useful in several aspects. First, it can be used to study the frequency and radiative forcing of thin water clouds, 
and, in addition, to study how they are influenced by anthropogenic activity (e.g. whether the aerosol loading in the atmosphere alters thin clouds properties). Second, this method can be used to study the cloud's "twilight zone", as comparison to standard optical depth measurements (either from space-borne platforms such as MODIS or from ground based instruments such as the AERONET) can be easily achieved. Third, it is possible to study the microphysical processes which control cloud's edges, as this method can retrieve their spatial and temporal characteristics. Finally, this method can be further expanded to study what seems as optically clear sky conditions, in a search for unique phenomena that could be spectrally analyzed.

A field campaign was conducted during the summers of 2010 and 2011, and the optical and microphysical properties of thin clouds were retrieved by the proposed method. The results of this campaign will be published in a following paper.

\section{Appendix A}

\section{Technical description of the SR5000}

The main instrumental device in this study was the SR5000 (CI-Systems, Israel), a calibrated spectro-radiometer in the range of 2.5-14 $\mu \mathrm{m}$ (Cabib et al., 2006). This passive remote sensing sensor employs a circular variable (interference) filter (CVF) to obtain the spectral radiance from the environment. The angular speed of the CVF is computer controllable and it determines the spectra acquisition rate of the sensor. The sensor's field of view (FOV) is selectable in the range of $0.5-6 \mathrm{mrad}$ and it affects the spectral resolution of the sensor, which varies from $1 \%$ to $2 \%$ of the central wavelength, depending on the FOV and spectral range. The radiometer was calibrated with an extended area blackbody (SR80, CISystems, Israel), that enables the sensor to provide the obtained spectra in units of radiance (Watts cm $\mathrm{cm}^{-2} \mathrm{str}^{-1} \mu \mathrm{m}^{-1}$ ). In addition, the sensor is equipped with an internal chopper which its temperature is monitored constantly. The chopper is coated by a thin layer that is characterized by a uniform, and close to unity emissivity. The radiance is chopped with the frequency of the chopper, and a lock in amplifier enhances the sensor's signal to noise (SNR) by reading the chopped signal within a small bandwidth. The spectroradiometer uses a "sandwich" LN2 cooled detector, which includes two attached detectors: an InSb detector with high sensitivity in the spectral region of $2.5-5.5 \mu \mathrm{m}$ and a MCT detector in the range of $5.5-14 \mu \mathrm{m}$. Throughout our measurement campaign, we have pointed the SR5000 to the zenith and operated it in a single mode: the FOV was selected to $6 \mathrm{mrad}$ and an acquisition rate of $0.5 \mathrm{~Hz}$ was applied. The noise equivalent spectral radiance (NESR) under this configuration was measured in the lab, and it stands on $6.4 \times 10^{-6} \mathrm{~W} \mathrm{~cm}^{-2} \mathrm{str}^{-1} \mu \mathrm{m}^{-1}$ for wavelength of $10 \mu \mathrm{m}$.
Acknowledgements. The authors are thankful to Amnon Sharon and Nadav Gilad for their essential technical support in conducting the validation experiment. This research was partly funded by the Israeli Ministry of Science and Technology (IMOS - Tashtiot) grant.

Edited by: B. Mayer

\section{References}

Agassi, E., Ronen, A., Shiloah, N., and Hirsch, E.: Discrimination between natural dense dust clouds with IR spectral measurements, Int. J. High Speed Elect. Syst., 18, 647-660, 2008.

Berk, A., Bernstein, L. S., and Robertson, D. C.: MODTRAN: A moderate resolution model for LOWTRAN 7, Tech. Rep. GLTR89-0122, Geophysics Laboratory, Air Force System Command, Hanscom AFB, Massachusetts, 1989.

Cabib, D., Gil, A., and Buckwald, R. A.: High performance spectroradiometer for very accurate radiometric calibrations and testing of blackbody sources and EO test equipment, Proc. SPIE 6207, 62070L, 2006.

Chiu, J. C., Huang, C. H., Marshak, A., Slutsker, I., Giles, D. M., Holben, B. N., Knyazikhin, Y., and Wiscombe, W. J.: Cloud optical depth retrievals from the Aerosol Robotic Network (AERONET) cloud mode observations, J. Geophys. Res., 115, D14202, doi:10.1029/2009JD013121, 2010.

Deirmendjian, D.: Scattering and Polarization Properties of Water Clouds and Hazes in the Visible and Infrared, Appl. Optics, 3, 187-196, 1964.

Frisch, S., Shupe, M., Djalalova, I., Feingold, G., and Poellot, M.: The Retrieval of Stratus Cloud Droplet Effective Radius with Cloud Radars, J. Atmos. Ocean. Tech., 19, 835-842, doi:10.1175/1520-0426(2002)019<0835:TROSCD > 2.0.CO;2, 2002.

Han, Y. and Westwater, E. R.: Remote Sensing of Tropospheric Water Vapor and Cloud Liquid Water by Integrated GroundBased Sensors, J. Atmos. Ocean. Tech., 12, 1050-1059, doi:10.1175/1520-0426(1995)012<1050:RSOTWV > 2.0.CO;2, 1995.

Hansen, J. E. and Travis, L. D.: Light scattering in planetary atmospheres, Space Sci. Rev., 16, 527-610, doi:10.1007/bf00168069, 1974.

Heintzenberg, J. and Charlson, R. J. (Eds.): Clouds in the perturbed climate system; their relationship to energy balance, atmospheric dynamics, and precipitation, The MIT Press, Cambridge, Massachusetts, 2009.

Hirsch, E. and Agassi, E.: Detection of gaseous plumes in IR hyperspectral images using hierarchical clustering, Appl. Optics, 46, 6368-6374, 2007

Holben, B. N., Eck, T. F., Slutsker, I., Tanré, D., Buis, J. P., Setzer, A., Vermote, E., Reagan, J. A., Kaufman, Y. J., Nakajima, T., Lavenu, F., Jankowiak, I., and Smirnov, A.: AERONET A Federated Instrument Network and Data Archive for Aerosol Characterization, Remote Sensing of Environment, 66, 1-16, doi:10.1016/s0034-4257(98)00031-5, 1998.

IPCC, 2007: Climate Change 2007: The Physical Science Basis, Contribution of Working Group I to the Fourth Assessment Report of the Intergovernmental Panel on Climate Change, edited by: Solomon, S., Qin, D., Manning, M., Chen, Z., Marquis, M., 
Averyt, K. B., Tignor, M., and Miller, H. L., Cambridge University Press, Cambridge, UK and New York, NY, USA, 995 pp., 2007.

Johnson, R. A. and Wichern, D. W.: Applied multivariate statistical analysis, 3rd Edn., Prentice-Hall International, Inc., USA, 1992.

King, M. D., Tsay, S.-C., Platnick, S. E., Wang, M., and Liou, K.N.: Cloud retrieval algorithms for MODIS: optical thickness, effective particle radius, and thermodynamic phase, MODIS Algorithm Theoretical Basis Document, MOD06 - Cloud product, No. ATBD-MOD-05, 1997.

King, M. D., Platnick, S., Yang, P., Arnold, G. T., Gray, M. A., Riedi, J. C., Ackerman, S. A., and Liou, K.-N.: Remote Sensing of Liquid Water and Ice Cloud Optical Thickness and Effective Radius in the Arctic: Application of Airborne Multispectral MAS Data, J. Atmos. Ocean. Tech., 21, 857-875, doi:10.1175/15200426(2004)021<0857:RSOLWA > 2.0.CO;2, 2004.

Köhler, H.: The nucleus in and the growth of hygroscopic droplets, Trans. Faraday Soc., 32, 1152-1161, 1936.

Koren, I., Remer, L. A., Kaufman, Y. J., Rudich, Y., and Martins, J. V.: On the twilight zone between clouds and aerosols, Geophys. Res. Lett., 34, L08805, doi:10.1029/2007g1029253, 2007.

Koren, I., Oreopoulos, L., Feingold, G., Remer, L. A., and Altaratz, O.: How small is a small cloud?, Atmos. Chem. Phys., 8, 38553864, doi:10.5194/acp-8-3855-2008, 2008.

Koren, I., Remer, L. A., Altaratz, O., Martins, J. V., and Davidi, A.: Aerosol-induced changes of convective cloud anvils produce strong climate warming, Atmos. Chem. Phys., 10, 5001-5010, doi:10.5194/acp-10-5001-2010, 2010

Kruse, F. A., Lefkoff, A. B., Boardman, J. W., Heidebrecht, K. B., Shapiro, A. T., Barloon, P. J., and Goetz, A. F. H.: The spectral image-processing system (SIPS) - interactive visualization and analysis of imaging spectrometer data, Remote Sens. Environ., 44, 145-163, 1993.

Liu, G., Shao, H., Coakley, J. A., Curry, J. A., Haggery, J. A., and Tschudi, M. A.: Retrieval of cloud droplet size from visible and microwave radiometric measurements during INDOEX: Implication to aerosols' indirect radiative effect, J. Geophys. Res., 108, 4006, doi:10.1029/2001JD001395, 2003.

Mätzler, C.: MATLAB Functions for Mie scattering and absorption, IAP Res. Rep. No. 02-08, Insitut für Angewandte, 2002.

Markowicz, K. M., Flatau, P. J., Vogelmann, A. M., Quinn, P. K., and Welton, E. J.: Clear-sky infrared aerosol radiative forcing at the surface and the top of the atmosphere, Q. J. Roy. Meteorol. Soc., 129, 2927-2947, doi:10.1256/qj.02.224, 2003.

McBride, P. J., Schmidt, K. S., Pilewskie, P., Kittelman, A. S., and Wolfe, D. E.: A spectral method for retrieving cloud optical thickness and effective radius from surface-based transmittance measurements, Atmos. Chem. Phys., 11, 7235-7252, doi:10.5194/acp-11-7235-2011, 2011.

McCaa, D. J. and Shaw, J. H.: The infrared spectrum of ozone, J. Mol. Spectrosc., 25, 374-397, doi:10.1016/s00222852(68)80050-5, 1968.

Mie, G.: Beiträge zur Optik trüber Medien, speziell kolloidaler Metallösungen, Ann. Physik, 330, 377-445, doi:10.1002/andp.19083300302, 1908.

Miles, N. L., Verlinde, J., and Clothiaux, E. E.: Cloud Droplet Size Distributions in Low-Level Stratiform Clouds, J. Atmos. Sci., 57, 295-311, doi:10.1175/15200469(2000)057<0295:cdsdil>2.0.co;2, 2000.
Nakajima, T. and King, M. D.: Determination of the Optical Thickness and Effective Particle Radius of Clouds from Reflected Solar Radiation Measurements, Part I: Theory, J. Atmos. Sci., 47, 1878-1893, doi:10.1175/15200469(1990)047<1878:DOTOTA > 2.0.CO;2, 1990.

Nilsson, B.: Meteorological influence on aerosol extinction in the 0.2?40- $\mu \mathrm{m}$ wavelength range, Appl. Optics, 18, 3457-3473, 1979.

Palik, E.: Handbook of Optical Constants of Solids, 5 Volume Set, Academic Press, 1997.

Park, B., Windham, W. R., Lawrence, K. C., and Smith, D. P.: Contaminant Classification of Poultry Hyperspectral Imagery using a Spectral Angle Mapper Algorithm, Biosyst. Eng., 96, 323-333, doi:10.1016/j.biosystemseng.2006.11.012, 2007.

Ramanathan, V., Cess, R. D., Harrison, E. F., Minnis, P., Barkstrom, B. R., Ahmad, E., and Hartmann, D.: Cloud-Radiative Forcing and Climate: Results from the Earth Radiation Budget Experiment, Science, 243, 57-63, doi:10.1126/science.243.4887.57, 1989.

Richwine, L. J., Clapp, M. L., Miller, R. E., and Worsnop, D. R.: Complex refractive indices in the infrared of nitric acid trihydrate aerosols, Geophys. Res. Lett., 22, 2625-2628, doi:10.1029/95gl02650, 1995.

Rodgers, C. D.: Inverse Methods for Atmospheric Sounding: Theory and Practice, vol. 2 of Series on Atmospheric, Oceanic and Planetary Physics, edited by: Taylor, F. W., World Scientific, 2000.

Rothman, L. S., Barbe, A., Benner, D. C., Brown, L. R., CamyPeyret, C., Carleer, M. R., Chance, K., Clerbaux, C., Dana, V., Devi, V. M., Fayt, A., Flaud, J. M., Gamache, R. R., Goldman, A., Jacquemart, D., Jucks, K. W., Lafferty, W. J., Mandin, J. Y., Massie, S. T., Nemtchinov, V., Newnham, D. A., Perrin, A., Rinsland, C. P., Schroeder, J., Smith, K. M., Smith, M. A. H., Tang, K., Toth, R. A., Vander Auwera, J., Varanasi, P., and Yoshino, K.: The HITRAN molecular spectroscopic database: edition of 2000 including updates through 2001, J. Quant. Spectrosc. Ra., 82, 5-44, 2003.

Slingo, A.: Sensitivity of the Earth's radiation budget to changes in low clouds, Nature, 343, 49-51, 1990.

Stephens, G. L.: Remote Sensing of the Lower Atmosphere, An Introduction, Oxford University Press, 523 pp., 1994.

Thomas, G. E., Bass, S. F., Grainger, R. G., and Lambert, A.: Retrieval of aerosol refractive index from extinction spectra with a damped harmonic-oscillator band model, Appl. Optics, 44, 1332-1341, 2005.

Toon, O. B., Pollack, J. B., and Khare, B. N.: The optical constants of several atmospheric aerosol species: ammonium sulfate, aluminum oxide, and sodium chloride, J. Geophys. Res., 81, 57335748, 1976.

Trenberth, K. E., Fasullo, J. T., and Kiehl, J.: Earth's Global Energy Budget, B. Am. Meteorol. Soc., 90, 311-323, doi:10.1175/2008bams2634.1, 2009.

Tselioudis, G. and Jakob, C.: Evaluation of midlatitude cloud properties in a weather and a climate model: Dependence on dynamic regime and spatial resolution, J. Geophys. Res., 107, 4781, doi:10.1029/2002jd002259, 2002.

Turner, D. D.: Arctic Mixed-Phase Cloud Properties from AERI Lidar Observations: Algorithm and Results from SHEBA, J. Appl. Meteorol., 44, 427-444, doi:10.1175/JAM2208.1, 2005. 
Turner, D. D., Vogelmann, A. M., Austin, R. T., Barnard, J. C., Cady-Pereira, K., Chiu, C., Clough, S. A., Flynn, C. J., Khaiyer, M. M., Liljegren, J. C., Johnson, K. L., Lin, B., Long, C. N., Marshak, A., Matrosov, S. Y., McFarlane, S. A., Miller, M. A., Min, Q., Minnis, P., O'Hirok, W., Wang, Z., and Wiscombe, W. J.: Thin Liquid Water Clouds: Their Importance and Our Challenge, B. Am. Meteorol. Soc., 88, 177-190, 2007.

Volz, F. E.: Infrared Refractive Index of Atmospheric Aerosol Substances, Appl. Optics, 11, 755-759, 1972.

Volz, F. E.: Infrared Optical Constants of Ammonium Sulfate, Sahara Dust, Volcanic Pumice, and Flyash, Appl. Optics, 12, 564568, 1973.

Weng, F. and Grody, N. C.: Retrieval of cloud liquid water using the special sensor microwave imager (SSM/I), J. Geophys. Res., 99, 25535-25551, doi:10.1029/94jd02304, 1994.

Wylie, D., Jackson, D. L., Menzel, W. P., and Bates, J. J.: Trends in Global Cloud Cover in Two Decades of HIRS Observations, J. Climate, 18, 3021-3031, doi:10.1175/JCLI3461.1, 2005.
Yamamoto, G., Tanaka, M., and Asano, S.: Radiative Transfer in Water Clouds in the Infrared Region, J. Atmos. Sci., 27, 282-292, doi:10.1175/15200469(1970)027<0282:RTIWCI>2.0.CO;2, 1970.

Yang, Y., Marshak, A., Chiu, J. C., Wiscombe, W., Palm, S. P., Davis, A. B., Spangenberg, D. A., Nguyen, L., Spinhirne, J. D., and Minnis, P.: Retrievals of thick cloud optical depth from the Geoscience Laser Altimeter System (GLAS) by calibration of solar background signal, J. Atmos. Sci., 65, 3513-3527, 2008.

Yuhas, R. H., Goetz, A. F. H., and Boardman, J. W.: Discrimination among semi-arid landscape endmembres using the spectral angle mapper (SAM) algorithm, in: Summaries of the 3rd annu. JPL Airborne Geosci. Workshop, edited by: Green, R. O., Publ. 9214, vol. 1, 147-149, 1992. 